\title{
Common Properties of Riemann Zeta Function, Bessel Functions and Gauss Function Concerning Their Zeros
}

\author{
Alfred Wünsche \\ Institut für Physik, Humboldt-Universität, MPG Nichtklassische Strahlung, Berlin, Germany \\ Email: alfred.wuensche@physik.hu-berlin.de
}

How to cite this paper: Wünsche, A. (2019) Common Properties of Riemann Zeta Function, Bessel Functions and Gauss Function Concerning Their Zeros. Advances in Pure Mathematics, 9, 281-316. https://doi.org/10.4236/apm.2019.93013

Received: March 1, 2019

Accepted: March 26, 2019

Published: March 29, 2019

Copyright $\odot 2019$ by author(s) and Scientific Research Publishing Inc. This work is licensed under the Creative Commons Attribution International License (CC BY 4.0).

http://creativecommons.org/licenses/by/4.0/

\section{(c) (i) Open Access}

\begin{abstract}
The behavior of the zeros in finite Taylor series approximations of the Riemann Xi function (to the zeta function), of modified Bessel functions and of the Gaussian (bell) function is investigated and illustrated in the complex domain by pictures. It can be seen how the zeros in finite approximations approach to the genuine zeros in the transition to higher-order approximation and in case of the Gaussian (bell) function that they go with great uniformity to infinity in the complex plane. A limiting transition from the modified Bessel functions to a Gaussian function is discussed and represented in pictures. In an Appendix a new building stone to a full proof of the Riemann hypothesis using the Second mean-value theorem is presented.
\end{abstract}

\section{Keywords}

Riemann Zeta and Xi Function, Modified Bessel Functions, Second Mean-Value Theorem or Gauss-Bonnet Theorem, Riemann Hypothesis

\section{Introduction}

The present paper tries to find out the common ground for the zeros of the Riemann zeta function $\zeta(z)=\zeta(x+\mathrm{i} y)$ and of the modified Bessel functions $\mathrm{I}_{v}(z)$ (or Bessel functions $\mathrm{J}_{v}(z)$ of imaginary argument $z$ ) for imaginary argument $z$ and, furthermore, for the absence of zeros of the Gaussian Bell function $\exp \left(z^{2}\right)$. For the function now called Riemann zeta function $\zeta(z)$ which was known already to Euler but was extended by Riemann to the complex plane Riemann expressed the hypothesis that all nontrivial zeros of this function lie on the axis $z=\frac{1}{2}+i y$ that means on the axis through $x=\frac{1}{2}$ and parallel to 
the imaginary axis $y$ (Riemann hypothesis) [1] [2] [3] (both with republication of Riemann's paper) and many others, e.g. [4] [5] [6] [7] [8]. Riemann never proved his hypothesis. He introduced in [1] also a Xi function $\xi(z)$ which excludes the only singularity of the function $\zeta(z)$ at $z=1$ and its trivial zeros at $z=-2,-4, \cdots$ and possesses more symmetry than the zeta function $\zeta(z)$. Concerning their zeros it is equivalent to the nontrivial zeros of the zeta function. In present paper we will mainly have to do only with this Xi function $\xi(z)$ which we displaced in a way that its zeros lie on the imaginary axis provided; the Riemann Hypothesis is correct and we denote as Xi function $\Xi(z)$. With respect to the position of the zeros the function $\Xi(z)$ is fully equivalent to the nontrivial zeros of the Riemann zeta function $\zeta(z)$ only with displacement of the imaginary axis to these zeros.

The content of this article was not intended as a proof of the Riemann hypothesis but during the work we found a further, as it seems, essential building stone for its proof by the second mean-value approach which is represented in Appendix. The article is merely intended as an illustration to the zeros of a function with a possible representation in an integral form given in Section 2 (Equation (2.8)) with monotonically decreasing functions $\Omega(u)$ satisfied by the Riemann Xi function and by the modified Bessel functions and explains why the Gauss bell function although it can be represented in such form does not possess zeros. Other kinds of interesting illustrations of the Riemann zeta function (and of other functions) by the Newton flow are given in [9] [10]. A main purpose was to understand how the zeros in the Taylor series approximations of such functions behave when we go from one order of the approximation to the next higher one. To get the possibility of a comparison with the pictures of zeros for functions without an integral representation of the mentioned form we made an analogous picture for an unorthodox entire function in Section 9.

\section{Basic Equations for the Considered Functions}

The Xi function $\Xi(z)$ to the Riemann zeta function $\zeta(z)$ is defined by

$$
\Xi(z) \equiv \xi\left(\frac{1}{2}+z\right),
$$

where $\zeta(z)$ is the Riemann Xi function [1] which is related to the Riemann zeta function $\zeta(z)$ by ${ }^{1}$

$$
\xi(z) \equiv(z-1)\left(\frac{z}{2}\right) ! \pi^{-\frac{z}{2}} \zeta(z) .
$$

The Riemann zeta function is basically defined by the following Euler product

$$
\zeta(z) \equiv \prod_{n=1}^{\infty}\left(1-\frac{1}{\left(p_{n}\right)^{2}}\right)^{-1}, \quad\left(p_{1}=2, p_{2}=3, p_{3}=5, p_{4}=7, \cdots\right),
$$

${ }^{1}$ Riemann denotes the complex variable by $s=\sigma+\mathrm{it}$ that is $\xi(s)$ for the Xi function and $\zeta(s)$ for the zeta function. 
where $p_{n}$ is the sequence of prime numbers beginning with $p_{1}=2$. The definition of the Riemann $\mathrm{Xi}$ function (2.3) is equivalent to the definition by the following (Dirichlet) series for complex variable $z=x+i y$

$$
\zeta(z)=\sum_{n=1}^{\infty} \frac{1}{n^{z}}, \quad(x=\operatorname{Re}(z)>1),
$$

which is convergent for $x>1$ and arbitrary $y$ and can be analytically continued to the whole complex $z$-plane. The function $\Xi(z)$ is an entire function which excludes the only singularity of $\zeta(z)$ at $z=1$ and its "trivial" zeros at $z=-2,-4,-6, \cdots$.

Next we consider the whole class of modified Bessel functions $I_{v}(z)$ of imaginary argument which is connected with the basic class of Bessel functions $\mathrm{J}_{v}(z)$ in the following slightly modified form by $(\mu ! \equiv \Gamma(\mu+1))$

$$
\left(\frac{2}{z}\right)^{v} \mathrm{I}_{v}(\mathrm{z})=\left(\frac{2}{\mathrm{i} z}\right)^{v} \mathrm{~J}_{v}(\mathrm{iz}) \equiv \sum_{m=0}^{\infty} \frac{1}{m !(m+v) !}\left(\frac{z}{2}\right)^{2 m}, \quad(v \in \mathbb{R}) .
$$

The functions $\left(\frac{2}{z}\right)^{v} I_{v}(z)$ are entire functions which satisfy the differential equation

$\left\{\left(z \frac{\partial}{\partial z}\right)^{2}+2 v z \frac{\partial}{\partial z}-z^{2}\right\}\left(\frac{2}{z}\right)^{v} I_{v}(z)=0 \Leftrightarrow\left\{\left(z \frac{\partial}{\partial z}\right)^{2}-z^{2}-v^{2}\right\} I_{v}(z)=0$.

In comparison to $I_{v}(z)$ the functions $\left(\frac{2}{z}\right)^{v} I_{v}(z)$ exclude the zeros or infinities of the first ones at $z=0$ but the other zeros remain the same for both functions.

Finally, we consider the Gaussian functions $\exp \left(\frac{a^{2} z^{2}}{4}\right)$ with parameter $a^{2}>0$ which can be represented by the following integral representation (continued from the imaginary axis $y$ to the whole complex $z$-plane)

$$
\exp \left(\frac{a^{2} z^{2}}{4}\right)=\frac{2}{\sqrt{\pi a^{2}}} \int_{0}^{+\infty} \mathrm{d} u \exp \left(-\frac{u^{2}}{a^{2}}\right) \operatorname{ch}(u z), \quad\left(a^{2}>0\right),
$$

which become Gaussian bell functions for imaginary argument $z=\mathrm{i} y$. Clearly, the functions $\exp \left(\frac{a^{2} z^{2}}{4}\right)$ do not possess zeros on the imaginary axis and zeros at all.

The three mentioned types of functions written as $\Xi(z)$ have in common that they are symmetrical functions in $z$ and that they possess a representation by an integral of the type

$$
\left.\Xi(z)=\int_{0}^{+\infty} \mathrm{d} u \Omega(u) \operatorname{ch}(u z)\right)=\Xi(-z)=\left(\Xi\left(z^{*}\right)\right)^{*}, \quad \Omega(u)=\Omega(-u),
$$

with monotonically decreasing functions $\Omega(u)$ for $0 \leq u \leq \infty$ that means

$$
0 \leq u_{1} \leq u_{2} \Rightarrow \Omega\left(u_{1}\right) \geq \Omega\left(u_{2}\right), \Omega(u \rightarrow+\infty)=0 .
$$


The Taylor series of $\Xi(z)$ can be written in the form

$$
\begin{gathered}
\Xi(z)=\sum_{m=0}^{\infty} \Omega_{2 m} z^{2 m}, \quad \Omega_{n} \equiv \frac{1}{n !} \int_{-\infty}^{+\infty} \mathrm{d} u \frac{1}{2}(\Omega(u)+\Omega(-u)) u^{n} \\
\Omega_{2 m}=\frac{1}{(2 m) !} \int_{0}^{+\infty} \mathrm{d} u \Omega(u) u^{2 m}, \quad \Omega_{2 m+1}=0, \quad(m=0,1,2, \cdots),
\end{gathered}
$$

where $\Omega_{n},(n=0,1,2, \cdots)$ are defined as the moments of the symmetrical function $\Omega(u)$ with respect to the reference point $u_{0}=0$. A consequence of the definitions in (2.8) and (2.10) is

$$
\Xi(0)=\int_{0}^{+\infty} \mathrm{d} u \Omega(u) \equiv \Omega_{0} .
$$

The odd moments $\Omega_{2 m+1}$ of the function $\Omega(u)$ in the definition (2.10) vanish. The function $\Xi(z)$ at $z=0$ is equal to the zeroth moment $\Omega_{0}$ of the function $\Omega(u)$ and is independent of the chosen reference point. In the following the moments of the function $\Omega(u)$ play an important role.

That $\Omega(u)$ is a symmetrical function in $u$ is, in principle, not necessary since the integration over $u$ in (2.8) is restricted by $u \geq 0$ but the symmetry permits to extend the integral over negative values of $u$ using it in the form

$$
\Xi(z)=\frac{1}{2} \int_{-\infty}^{+\infty} \mathrm{d} u \Omega(u) \mathrm{e}^{u z}=\frac{1}{2} \int_{-\infty}^{+\infty} \mathrm{d} u \Omega(u) \operatorname{ch}(u z)+\underbrace{\frac{1}{2} \int_{-\infty}^{+\infty} \mathrm{d} u \Omega(u) \operatorname{sh}(u z),}_{=0}
$$

which for imaginary $z=\mathrm{i} y$ is a Fourier transformation of $\Omega(u)$ with the inversion

$$
\Omega(u)=\frac{1}{\pi} \int_{-\infty}^{+\infty} \mathrm{d} y \Xi(\mathrm{i} y) \mathrm{e}^{-\mathrm{i} u y},
$$

if the integral exists in some sense (e.g. weak convergence). We consider this now more explicitly.

The explicit representation of the Xi function $\Xi(z)$ to the Riemann Xi function $\xi(z)=\Xi\left(z-\frac{1}{2}\right)$ in this form (2.8) together with (2.9) is

$$
\begin{gathered}
\Xi(z)=\int_{0}^{+\infty} \mathrm{d} u \Omega(u) \operatorname{ch}(u z), \\
\Omega(u) \equiv 4 \exp \left(\frac{u}{2}\right) \sum_{n=1}^{\infty} \pi n^{2} \mathrm{e}^{2 u}\left(2 \pi n^{2} \mathrm{e}^{2 u}-3\right) \exp \left(-\pi n^{2} \mathrm{e}^{2 u}\right)=\Omega(-u),
\end{gathered}
$$

with the special values

$$
\begin{gathered}
\Xi(0)=\int_{0}^{+\infty} \mathrm{d} u \Omega(u) \approx 0.4971207782, \quad \Xi\left( \pm \frac{1}{2}\right)=\frac{1}{2}, \\
\Omega(0) \approx 1.7867876019 .
\end{gathered}
$$

This was derived in detail in [11]. The function $\Omega(u)$ together with its first derivative $\Omega^{(1)}(u)$ is represented in Figure 1 .

The function $\Omega(u)$ is monotonically decreasing from $u=0$ on and decreases rapidly for $u \rightarrow \pm \infty$. The function $\Xi(z)$ increases rapidly for real $z=x \rightarrow \pm \infty$ and decreases rapidly for imaginary $z=\mathrm{i} y \rightarrow \pm \mathrm{i} \infty$. The symmetry 

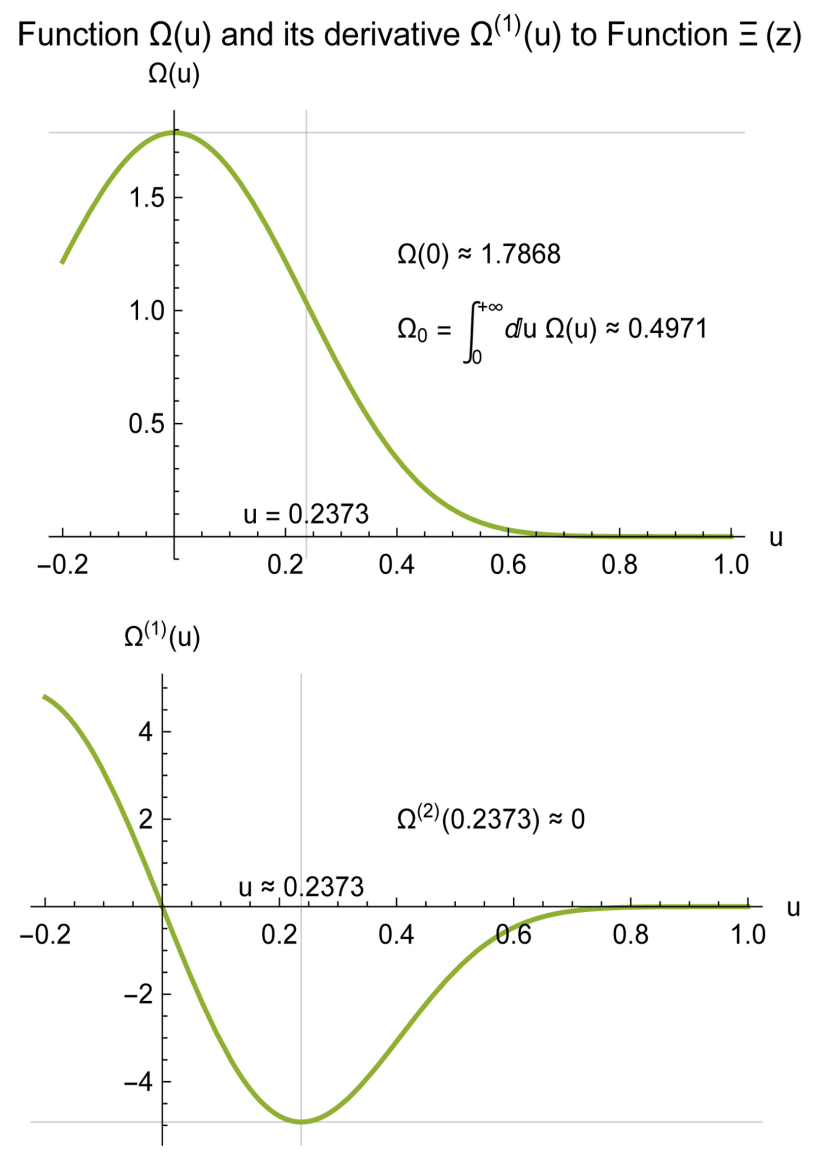

Figure 1. Representation of $\Omega(u)$ and of its first derivative $\Omega^{(1)}(u)$. The function $\Omega(u)$ is monotonically decreasing for $u \geq 0$ and together with its derivatives vanishes rapidly for $u \rightarrow+\infty$.

of $\Omega(u)$ is not easily to see from (2.14) and it was a genuine surprise for us to meet such a kind of a symmetrical function (see discussion in [11]). The function $\exp \left(-\frac{u}{2}\right) \Omega(u)$, for example, is already not a symmetrical function for $u \leftrightarrow-u$.

In case of the modified Bessel functions of imaginary argument the following basic integral representations are known which for the functions proportional to $\left(\frac{2}{z}\right)^{v} \mathrm{I}_{v}(z)$ may be written as follows (taking into account

$$
\begin{aligned}
\left(\frac{1}{2}\right) !=\frac{1}{2}\left(-\frac{1}{2}\right) != & \left.\frac{\sqrt{\pi}}{2}\right) \\
\Xi_{v}(z) & \equiv \Omega_{v}(0) \int_{0}^{1} \mathrm{~d} u\left(1-u^{2}\right)^{v-\frac{1}{2}} \operatorname{ch}(u z) \\
& =\Omega_{v}(0)\left(\frac{1}{2}\right) !\left(v-\frac{1}{2}\right) !\left(\frac{2}{z}\right)^{v} \mathrm{I}_{v}(z), \quad\left(v>-\frac{1}{2}\right),
\end{aligned}
$$

or expressed by the Confluent Hypergeometric function ${ }_{1} \mathrm{~F}_{1}(a ; c ; z)$ 


$$
\Xi_{v}(z)=\Omega_{v}(0) \frac{\left(\frac{1}{2}\right) !\left(v-\frac{1}{2}\right) !}{v !} \mathrm{e}^{\mp z}{ }_{1} \mathrm{~F}_{1}\left(v+\frac{1}{2} ; 2 v+1 ; \pm 2 z\right) .
$$

The Taylor series expansion is

$$
\Xi_{v}(z)=\Omega_{v}(0) \frac{\left(\frac{1}{2}\right) !\left(v-\frac{1}{2}\right) !}{v !}\left\{1+\sum_{m=1}^{\infty} \frac{v !}{m !(v+m) !}\left(\frac{z}{2}\right)^{2 m}\right\} .
$$

The functions $\Xi_{v}(z)$ possess the principal form

$$
\Xi_{v}(z)=\int_{0}^{+\infty} \mathrm{d} u \Omega_{v}(u) \operatorname{ch}(u z), \quad\left(v>-\frac{1}{2}\right),
$$

with the following explicit expressions for $\Omega_{v}(u)$

$$
\Omega_{v}(u)=\Omega_{v}(0)\left(1-u^{2}\right)^{v-\frac{1}{2}} \theta\left(1-u^{2}\right)=\Omega_{v}(-u),
$$

where $\theta(x)$ is Heaviside's step function defined by

$$
\theta(x) \equiv \begin{cases}1, & (x>0) \\ 0, & (x<0)\end{cases}
$$

It restricts the upper limit of integration in (2.19) according to the choice in (2.20) to $u=1$. The functions $\Omega_{v}(u)$ are equivalent to functions of $u^{2}$ only and are in this sense symmetrical functions of $u$ and for real $u^{2}<1$ we have to choose the real value of $\left(1-u^{2}\right)^{v-\frac{1}{2}}$ in case of non-integer $v-\frac{1}{2}$.

The first six cases of the function $\Xi_{v}(z)$ with integer or semi-integer index $v$ are

$$
\begin{array}{ll}
\Xi_{0}(z)=\Omega_{0}(0) \frac{\pi \mathrm{I}_{0}(z)}{2}, & \Xi_{\frac{1}{2}}(z)=\Omega_{\frac{1}{2}}(0) \frac{\operatorname{sh}(z)}{z}, \\
\Xi_{1}(z)=\Omega_{1}(0) \frac{\pi \mathrm{I}_{1}(z)}{2 z}, & \Xi_{\frac{3}{2}}(z)=\Omega_{\frac{3}{2}}(0) \frac{2(z \operatorname{ch}(z)-\operatorname{sh}(z))}{z^{3}}, \\
\Xi_{2}(z)=\Omega_{2}(0) \frac{3 \pi \mathrm{I}_{2}(z)}{2 z^{2}}, & \Xi_{\frac{5}{2}}(z)=\Omega_{\frac{5}{2}}(0) \frac{8\left(\left(3+z^{2}\right) \operatorname{sh}(z)-3 z \operatorname{ch}(z)\right)}{z^{5}} .
\end{array}
$$

The function $\Xi_{1}(z)$ does not exist since the corresponding integral in (2.16) is divergent or $\left(v-\frac{1}{2}\right)$ ! is not finite for $v=-\frac{1}{2}$ but

$\lim _{v \rightarrow-\frac{1}{2}} \frac{\Xi_{-\frac{1}{2}}(z)}{\left(v-\frac{1}{2}\right) !}=\frac{\operatorname{ch}(z)}{2}$ exists. Furthermore, we have introduced in

amplitudes $\Omega_{v}(0)$ and we will soon see that it is favorable to choose them for our purpose as constant for the whole class of functions $\Xi_{v}(z)$. We illustrate the functions $\Omega_{v}(u)$ and the corresponding functions $\Xi_{v}(z)$ with a certain important modification in next Section 3.

It is well known that the Bessel functions $\mathrm{J}_{v}(z)$ possess zeros only on the 
real axis $x$ and, correspondingly, $\mathrm{I}_{v}(z)$ only on the imaginary axis $y$. For this there exists a direct proof using their differential equations and which is similar to the derivation of duality and orthogonality relations (e.g. [12] [13]).

The considered Omega functions $\Omega(u)$ for the Riemann Xi function and $\Omega_{v}(u)$ for the modified Bessel functions and also for the Gaussian bell function have in common that they are monotonically decreasing functions for $u \geq 0$ up to $\Omega(+\infty)=0$.

\section{Modified Bessel Functions with Stretched Argument of the Kernel Function and Limiting Transition to Gaussian Bell Function}

We now calculate the even moments $\Omega_{v, 2 m}$ of the functions $\Omega_{v}(u)$ in (2.20) which lead to well-known integrals

$$
\begin{aligned}
\Omega_{v, 2 m} & \equiv \frac{\Omega_{v}(0)}{(2 m) !} \int_{0}^{+\infty} \mathrm{d} u \Omega_{v}(u) u^{2 m}=\frac{1}{(2 m) !} \int_{0}^{1} \mathrm{~d} u\left(1-u^{2}\right)^{v-\frac{1}{2}} u^{2 m} \\
& =\Omega_{v}(0) \frac{\left(v-\frac{1}{2}\right) !\left(m-\frac{1}{2}\right) !}{2(2 m) !(v+m) !}=\Omega_{v}(0) \frac{\left(\frac{1}{2}\right) !\left(v-\frac{1}{2}\right) !}{m !(v+m) ! 2^{2 m}}, \quad\left(\left(\frac{1}{2}\right) !=\frac{\sqrt{\pi}}{2}\right),
\end{aligned}
$$

and in the special case $m=0$ of zeroth moments

$$
\Omega_{v, 0}=\Omega_{v}(0) \frac{\left(\frac{1}{2}\right) !\left(v-\frac{1}{2}\right) !}{v !} .
$$

These are the areas under the curves $\Omega_{v}(u)$ on the positive $u$-axes. The following considerations show that it is favorable to make $\Omega_{v, 0}$ equal independently on parameter $v$ and to keep $\Omega_{v}(0)$ constant and we choose

$$
\Omega_{v}(0)=1 \text {. }
$$

To keep in addition also $\Omega_{v, 0}$ constant we have now the only possibility to introduce a stretch factor to the variable $u$ and we make the transformation (now already with choice (3.3))

$$
\Omega_{v}(u) \rightarrow \bar{\Omega}_{v}(u)=\left(1-\frac{u^{2}}{u_{v, 0}^{2}}\right)^{v-\frac{1}{2}} \theta\left(1-\frac{u^{2}}{u_{v, 0}^{2}}\right)=\Omega_{v}\left(\frac{u}{u_{v, 0}}\right),
$$

with the definition of $u_{v, 0}$

$$
u_{v, 0} \equiv \frac{v !}{\left(\frac{1}{2}\right) !\left(v-\frac{1}{2}\right) !} u_{\frac{1}{2}, 0}=\frac{u_{\frac{1}{2}, 0}}{\Omega_{v, 0}}, \quad u_{\frac{1}{2}, 0}=1,
$$

from which follows for the zeroth moments of $\bar{\Omega}_{v}(u)$

$$
\bar{\Omega}_{v, 0} \equiv \int_{0}^{+\infty} \mathrm{d} u \Omega_{v}(u)=\int_{0}^{u_{v, 0}} \mathrm{~d} u\left(1-\frac{u^{2}}{u_{v, 0}^{2}}\right)^{v-\frac{1}{2}}=1 .
$$

The functions $\bar{\Omega}_{v}(u)$ are graphically represented in Figure 2 for some low 


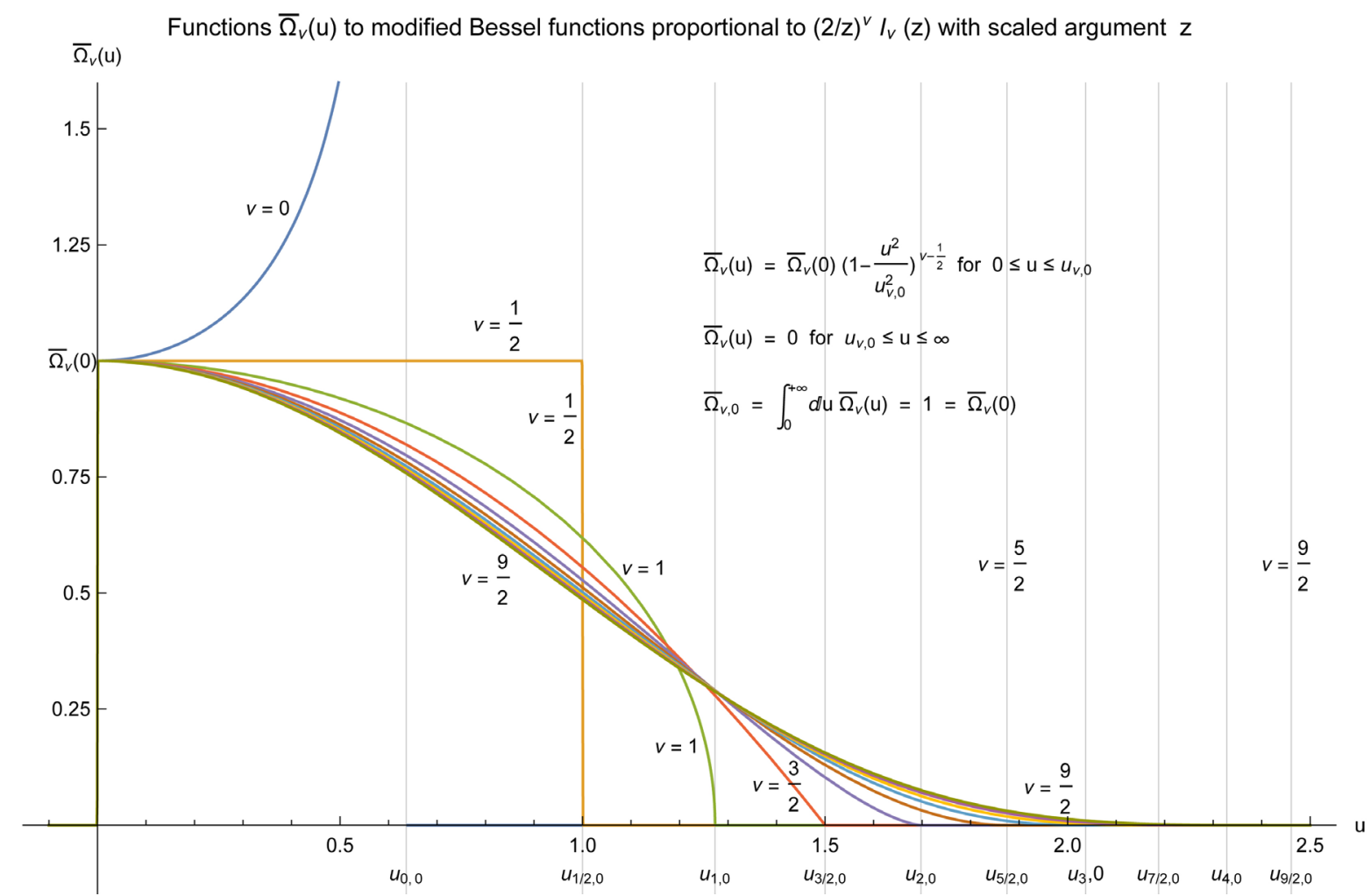

Figure 2. Functions $\bar{\Omega}_{v}(u)$ with stretched variable $u \rightarrow \frac{u}{u_{v, 0}}$ in comparison to functions $\Omega_{v}(u)$. G rid lines are set at the values $u_{v, 0}$ where the finite functions $\bar{\Omega}_{v}(u)$ end. In the chosen scale the curve $\bar{\Omega}_{v}(u)$ for $v=\frac{9}{2}$ is visibly already hardly to distinguish from a Gaussian bell function $\exp \left(-\frac{\pi u^{2}}{4}\right)$ and, therefore, is not drawn here.

values of $v$ up to $v=\frac{9}{2}$.

The corresponding transformation of the functions $\Xi_{v}(z)$ using (2.16) and (3.3) are

$$
\begin{aligned}
\Xi_{v}(z) \rightarrow \bar{\Xi}_{v}(z) & =u_{v, 0} \int_{0}^{u_{v, 0}} \frac{\mathrm{d} u}{u_{v, 0}}\left(1-\frac{u^{2}}{u_{v, 0}^{2}}\right)^{v-\frac{1}{2}} \operatorname{ch}\left(\frac{u}{u_{v, 0}} u_{v, 0} z\right) \\
& =u_{v, 0} \Xi_{v}\left(u_{v, 0} z\right)=v !\left(\frac{2}{u_{v, 0} z}\right)^{v} \mathrm{I}_{v}\left(u_{v, 0} z\right)
\end{aligned}
$$

Their Taylor series are

$$
\begin{aligned}
\bar{\Xi}_{v}(z) & =\sum_{m=0}^{\infty} \frac{v !}{m !(v+m) !}\left(\frac{u_{v, 0} z}{2}\right)^{2 m} \\
& =1+\sum_{m=1}^{\infty} \frac{v !}{m !(v+m) !}\left(\frac{v !}{\left(\frac{1}{2}\right) !\left(v-\frac{1}{2}\right) !} \frac{z}{2}\right)^{2 m}=\bar{\Xi}_{v}(-z),
\end{aligned}
$$


or with substitution $\quad z=\mp i y \quad$ (see also (2.5))

$$
\begin{aligned}
\bar{\Xi}_{v}(\mp \mathrm{i} y) & =v !\left(\frac{2}{u_{v, 0} y}\right)^{v} \mathrm{~J}_{v}\left(u_{v, 0} y\right) \\
& =1+\sum_{m=1}^{\infty} \frac{(-1)^{m} v !}{m !(v+m) !}\left(\frac{v !}{\left(\frac{1}{2}\right) !\left(v-\frac{1}{2}\right) !} \frac{y}{2}\right)^{2 m} .
\end{aligned}
$$

The first three functions $\bar{\Xi}_{v}(z)$ with integer and with semi-integer $v$ are explicitly

$$
\begin{aligned}
& \bar{\Xi}_{0}(z)=\mathrm{I}_{0}\left(\frac{2 z}{\pi}\right), \quad \bar{\Xi}_{\frac{1}{2}}(z)=\frac{\operatorname{sh}(z)}{z}, \\
& \bar{\Xi}_{1}(z)=\frac{\pi}{2 z} \mathrm{I}_{1}\left(\frac{4 z}{\pi}\right), \quad \bar{\Xi}_{\frac{3}{2}}(z)=\frac{4\left(3 z \operatorname{ch}\left(\frac{3 z}{2}\right)-2 \operatorname{sh}\left(\frac{3 z}{2}\right)\right)}{9 z^{3}}, \\
& \bar{\Xi}_{2}(z)=\frac{9 \pi^{2}}{32 z^{2}} I_{2}\left(\frac{16 z}{3 \pi}\right), \quad \bar{\Xi}_{\frac{5}{2}}(z)=\frac{512\left(\left(64+75 z^{2}\right) \operatorname{sh}\left(\frac{15 z}{8}\right)-120 z \operatorname{ch}\left(\frac{15 z}{8}\right)\right)}{16875 z^{5}} .
\end{aligned}
$$

The functions $\bar{\Xi}_{v}(z)$ on the imaginary axes $z=\mathrm{i} y$ for integer and semi-integer index $v$ up to $v=\frac{9}{2}$ are illustrated in Figure 3.

Figure 2 and Figure 3 admit the conjecture that $\bar{\Omega}_{v}(u)$ and $\bar{\Xi}_{v}$ (iy) for real variable $y$ become Gaussian bell functions in the limit $v \rightarrow \infty$. For $v=\frac{9}{2}$ in Figure 2 the function $\bar{\Omega}_{v}(u)$ is in visible way already hardly to distinguish from the Gaussian bell function (below we see to $\exp \left(-\frac{\pi u^{2}}{4}\right)$ ). That the mentioned approach for $v \rightarrow \infty$ to a Gaussian bell function is really true we establish exactly and determine these limits.

We now show that the new functions $\bar{\Xi}_{v}(z)$ in (3.7) for $v \rightarrow \infty$ go to a Gaussian function that becomes a Gaussian bell function for imaginary $z=i y$. As auxiliary formulae for $\frac{v !}{\left(v-\frac{1}{2}\right) !}$ in case of $v \gg 1$ follows

$$
\begin{aligned}
& \underbrace{\frac{v !}{\left(v-\frac{1}{2}\right)} !}_{\equiv \varphi(v)} \frac{\left(v-\frac{1}{2}\right) !}{(v-1) !}=v \approx \sqrt{v+\frac{1}{4}} \sqrt{v-\frac{1}{4}} \Rightarrow \frac{v !}{\left(v-\frac{1}{2}\right)} \approx \sqrt{v+\frac{1}{4}} \rightarrow \sqrt{v} \text {, } \\
& \frac{v !}{\left(\frac{1}{2}\right) !\left(v-\frac{1}{2}\right) !} \approx \frac{2}{\sqrt{\pi}} \sqrt{v+\frac{1}{4}} \approx \frac{2}{\sqrt{\pi}} \sqrt{v}, \quad\left(\left(\frac{1}{2}\right) !=\frac{\sqrt{\pi}}{2}\right),
\end{aligned}
$$




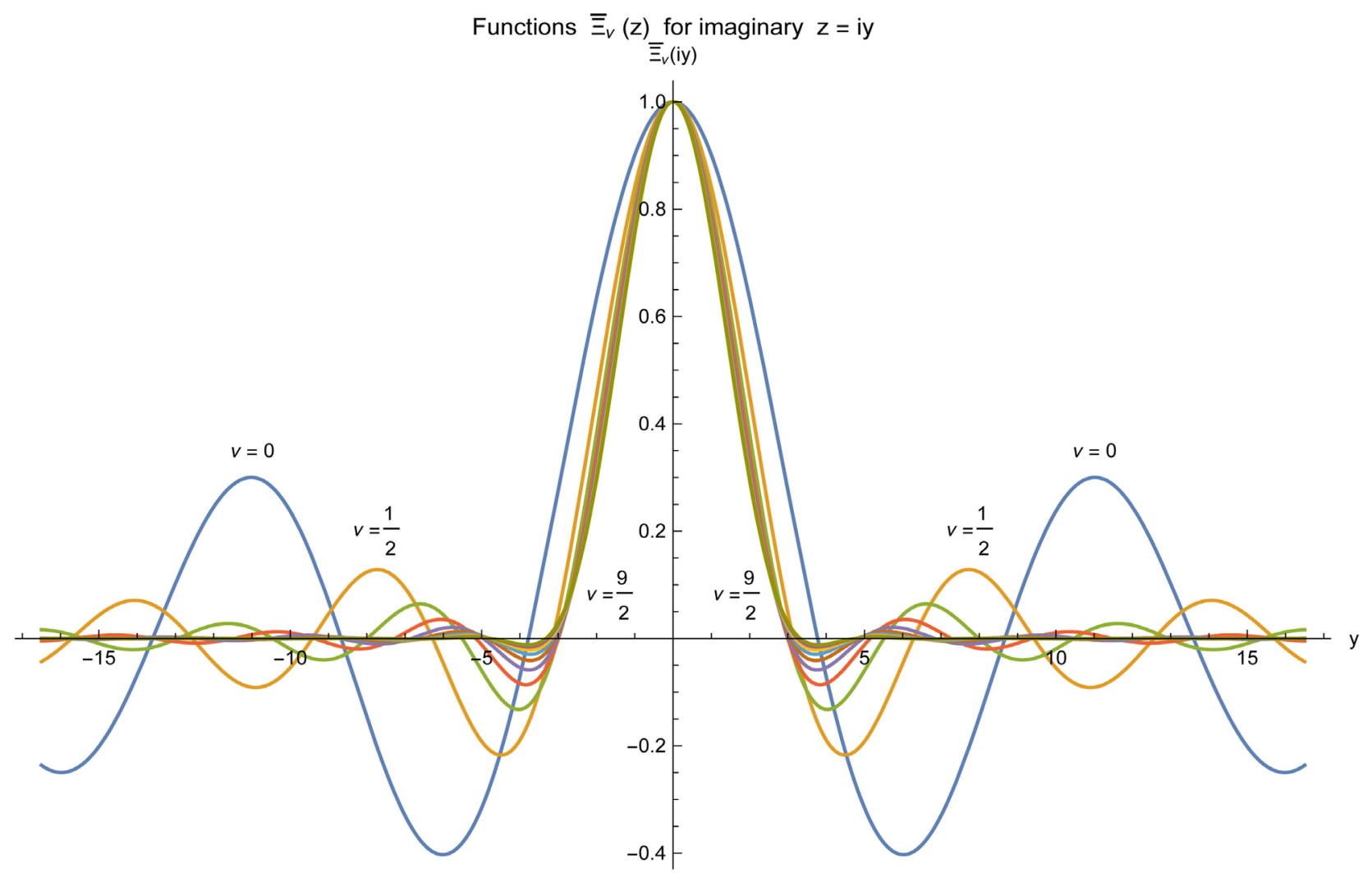

Figure 3. Functions $\bar{\Xi}_{v}\left(\right.$ iy) for values of $v$ up to $v=\frac{9}{2}$. W ith increasing $v$ from a certain $v$ on the first zeros of $\bar{\Xi}_{v}(z)$ increase (see also text) and the amplitudes of the oscillations decrease and in the limiting case $v \rightarrow+\infty$ they vanish and the resulting Gaussian function $\exp \left(-\frac{y^{2}}{\pi}\right)$ (on the imaginary $y$-axis $z=i y$ ) does not possess zeros. This Gaussian curve is not well to distinguish in the bulk of the other curves and is not drawn here.

and analogously for $v \gg 1$

$$
\frac{v !}{(v+m) !}=\frac{1}{(v+1)(v+2) \cdots(v+m)}=\frac{1}{v^{m}\left(1+\frac{1}{v}\right)\left(1+\frac{2}{v}\right) \cdots\left(1+\frac{m}{v}\right)} \approx v^{-m} .
$$

Applying these approximations we find from (3.8)

$$
\begin{aligned}
\lim _{v \rightarrow \infty} \bar{\Xi}_{v}(z) & =\lim _{v \rightarrow \infty} \sum_{m=0}^{\infty} \frac{v !}{m !(v+m) !}\left(\frac{v !}{\left(\frac{1}{2}\right) !\left(v-\frac{1}{2}\right) !} \frac{z}{2}\right)^{2 m} \\
& =\lim _{v \rightarrow \infty} \sum_{m=0}^{\infty} \frac{v^{-m}}{m !}\left(\sqrt{v} \frac{2}{\sqrt{\pi}} \frac{z}{2}\right)^{2 m}=\sum_{m=0}^{\infty} \frac{1}{m !}\left(\frac{z^{2}}{\pi}\right)^{m} \\
& =\exp \left(\frac{z^{2}}{\pi}\right) .
\end{aligned}
$$

This means that in the limiting transition $v \rightarrow \infty$ the functions $\bar{\Xi}_{v}(z)$ in (3.7) approach to a Gaussian function according to 


$$
\lim _{v \rightarrow \infty} v !\left(\frac{2\left(\frac{1}{2}\right) !\left(v-\frac{1}{2}\right) !}{v ! z}\right)^{v} \mathrm{I}_{v}\left(\frac{v !}{\left(\frac{1}{2}\right) !\left(v-\frac{1}{2}\right) !} \frac{z}{2}\right)=\exp \left(\frac{z^{2}}{\pi}\right)
$$

or by substitution $z=\mathrm{i} y$ with, in general, complex variable $y$

$$
\lim _{v \rightarrow \infty} v !\left(\frac{2\left(\frac{1}{2}\right) !\left(v-\frac{1}{2}\right) !}{v ! y}\right)^{v} \mathrm{~J}_{v}\left(\frac{v !}{\left(\frac{1}{2}\right) !\left(v-\frac{1}{2}\right) !} \frac{y}{2}\right)=\exp \left(-\frac{y^{2}}{\pi}\right)
$$

where we use the identity $2\left(\frac{1}{2}\right) !=\left(-\frac{1}{2}\right) !=\sqrt{\pi}$. From the corresponding limiting transition $\lim _{v \rightarrow \infty} \bar{\Omega}_{v}(u)$ using the definition in (3.4) we find

$\lim _{v \rightarrow \infty}\left(1-\left(\frac{\left(\frac{1}{2}\right) !\left(v-\frac{1}{2}\right) !}{v !}\right)^{2} u^{2}\right)^{v-\frac{1}{2}} \theta\left(1-\left(\frac{\left(\frac{1}{2}\right) !\left(v-\frac{1}{2}\right) !}{v !}\right)^{2} u^{2}\right)=\exp \left(-\frac{\pi u^{2}}{4}\right)$

We may check the transition from $\Omega(u)=\exp \left(-\frac{\pi u^{2}}{4}\right)$ to $\Xi(z)=\exp \left(\frac{z^{2}}{\pi}\right)$ via (2.8) using the auxiliary formula (2.7) with $a^{2}=\frac{4}{\pi}$.

Furthermore, we find using the approximation (3.11) that the factor between $u_{\frac{1}{2}, 0}$ and $u_{v, 0}$ in (3.5) approaches for $v \gg 1$ according to

$$
\frac{u_{v, 0}}{u_{\frac{1}{2}, 0}}=\frac{v !}{\left(\frac{1}{2}\right) !\left(v-\frac{1}{2}\right) !} \approx \frac{2}{\sqrt{\pi}} \sqrt{v+\frac{1}{4}}, \quad(v \gg 1),
$$

with a high precision and monotonically increasing without a finite limit.

We now show that for high increasing $v \gg 1$ the first positive roots of $\bar{\Xi}_{v}(\mathrm{iy})$ and thus also the higher roots increase. For $\bar{\Xi}_{v}(\mathrm{i} y)$ this is clear since it is proportional to $\mathrm{J}_{v}(y)$ and it is well known that their roots $y_{v, s},(s=1,2, \cdots)$ are situated approximately at

$$
y_{v, s}=\left(s+\frac{v}{2}-\frac{1}{4}\right) \pi-\cdots .
$$

However in $\bar{\Xi}_{v}(\mathrm{i} y)$ the argument in the Bessel functions is stretched by the factor $u_{v, 0}$ and therefore on the imaginary axis we have the functions $\mathrm{J}_{v}\left(u_{v, 0} y\right)$ that diminishes the values for the roots (3.18) by the factor $u_{v, 0}^{-1}$ and with the approximation (3.17) the zeros of $\bar{\Xi}_{v}(\mathrm{i} y)$ are now situated approximately at

$$
\frac{y_{v, s}}{u_{v, 0}} \approx \frac{\left(s+\frac{v}{2}-\frac{1}{4}\right) \pi^{\frac{3}{2}}}{\sqrt{4 v+1}} .
$$


Thus the first roots $(s=1)$ of $\bar{\Xi}_{v}$ (iy) go with $v \rightarrow \infty$ proportional to $\sqrt{v}$ also to infinity. This explains more in detail why the first zeros $(s=1)$ of the curves for $\bar{\Xi}_{v}(\mathrm{i} y)$ in Figure 3 go to the limiting case $\exp \left(-\frac{y^{2}}{\pi}\right)$ for $v \rightarrow \infty$.

We remind that the starting point for the derivation of the approximations was making equal the zeroth moments $\bar{\Omega}_{v, 0}$ by stretching the argument of the functions $\Omega_{v}(u)$ to new functions $\bar{\Omega}_{v}(u)$ leaving constant the amplitudes $\Omega_{v}(0)$. The zeros of $\bar{\Omega}_{v}(u)$ for $v \rightarrow \infty$ move in this process also to infinity but "very slowly". This is a good illustration why the Gaussian function $\exp \left(\frac{z^{2}}{\pi}\right)$ which on the imaginary axis $y$ becomes a Gaussian bell function $\exp \left(-\frac{y^{2}}{\pi}\right)$ does not possess zeros at all although its Omega function $\Omega(u)=\exp \left(-\frac{\pi u^{2}}{4}\right)$ is monotonically decreasing. The first zero and in this way all other zeros are moved by the limiting transition to infinity although very slowly. The same is the case with the discontinuities of derivatives in the functions $\bar{\Omega}_{v}(u)$.

\section{Graphical Representations to Zeros of the Xi Function to Riemann Zeta Function in Approximations by Truncated Taylor Series}

In this Section we consider the Taylor series expansions of the $\mathrm{Xi}$ function $\Xi(z)$ to the Riemann Xi function $\xi(s)$ in powers of $z$ defined in (2.14) according to

$$
\begin{gathered}
\Xi(z)=\int_{0}^{+\infty} \mathrm{d} u \Omega(u) \operatorname{ch}(u z) \\
=\sum_{m=0}^{\infty} \Omega_{2 m} z^{2 m}=\sum_{m=0}^{\infty} \frac{\Xi^{(2 m)}(0)}{(2 m) !} z^{2 m}, \\
\Omega_{2 m} \equiv \frac{1}{(2 m) !} \int_{0}^{+\infty} \mathrm{d} u \Omega(u) u^{2 m}=\frac{\Xi^{(2 m)}(0)}{(2 m) !} .
\end{gathered}
$$

The coefficients in this Taylor series are the even moments $\Omega_{2 m}$ of the function $\Omega(u)$ as defined.

We truncate the Taylor series of $\Xi(z)$ at upper summation numbers $m=M$

$$
\Xi_{2 M}(z)=\sum_{m=0}^{M} \Omega_{2 m} z^{2 m},
$$

and calculate all zeros of these approximations up to a certain maximal $M$ and make graphical representations of their zeros. As mentioned the coefficients of the series are determined by the even moments $\Omega_{2 m}$ of the function $\Omega(u)$ where the odd moments vanish automatically if we determine $\Omega(u)$ as 
symmetrical functions according to (2.8) and (2.10). In the following Sections we discuss the same also for modified Bessel functions $\mathrm{I}_{v}(\mathrm{z})$ (in particular for $v=\frac{1}{2}$ ) and for the limiting transition to a Gaussian function and compare this with the zeros for the Xi function (2.14) to the Riemann zeta function. In each approximation $\Xi_{2 M}(z)$ of the Taylor series we find $2 M$ complex zeros. It was very interesting to see which difference appears between the zeros on the imaginary axis and the bulk of the other zeros with increasing number $M$. This is best seen in graphical representations of the zeros.

For the function $\Omega(u)$ related to the Riemann hypothesis which is explicitly given in (2.14) we calculated this series numerically up to $2 M=100$ with sufficiently high precision and obtained the following numerical coefficients (we write here explicitly down only a few of the obtained coefficients) $)^{2}$

$$
\begin{aligned}
\Xi(z)= & 0.497120778188314+1.14859721575727 \times 10^{-2} z^{2} \\
& +1.23452018070318 \times 10^{-4} z^{4}+8.32355481385527 \times 10^{-7} z^{6} \\
& +3.99222655134413 \times 10^{-9} z^{8}+\cdots+1.05272334981972 \times 10^{-68} z^{48} \\
& +4.68273888631736 \times 10^{-72} z^{50}+1.97190495510121 \times 10^{-75} z^{52} \\
& +7.87602897895070 \times 10^{-79} z^{54}+2.98910175366638 \times 10^{-82} z^{56} \\
& +1.07971892491186 \times 10^{-85} z^{58}+3.71787541158066 \times 10^{-89} z^{60} \\
& +1.22215971011433 \times 10^{-92} z^{62}+\cdots+3.09393689867254 \times 10^{-158} z^{98} \\
& +5.11093592293365 \times 10^{-162} z^{100}+\cdots
\end{aligned}
$$

It is interesting to mention that the coefficients $(2 m) ! \Omega_{2 m},(m=1,2, \cdots)$ possess an absolute minimum for $m=21$ with $(42) ! \Omega_{42}(0)=1.1837577 \times 10^{-7}$ and, apparently, are ("slowly") monotonically increasing for $m>21=42$.

The first term in (4.3) can also be written (see [11])

$$
\begin{aligned}
& 0.497120778188313661 \\
& \approx \Xi(0)=\frac{1}{2}-\int_{1}^{+\infty} \frac{\mathrm{d} t}{2 \sqrt{t}} \sum_{n=1}^{\infty} \exp \left(-\pi n^{2} t^{2}\right) \\
& =\frac{1}{2}-\int_{1}^{+\infty} \mathrm{d} s \sum_{n=1}^{\infty} \exp \left(-\pi n^{2} s^{4}\right) \\
& \approx 0.5-0.002879221811686339
\end{aligned}
$$

corresponding to the splitting of terms in the representation (2.14) of $\Xi(z)$ after the substitution $\mathrm{e}^{u}=t=s^{2}$.

In the truncation of the series in powers of $z$ with the highest term proportional to $z^{2 M}$ we found by numerical solution of the corresponding algebraic equations of degree $2 M$ the $2 M$ complex solutions from which the

\footnotetext{
"We made the calculations two times with "Mathematica 3" (up to $2 \mathrm{M}=80$ and 18 digits) and with "Mathematica 6" (up to $2 \mathrm{M}=100$ and 20 digits) with a time difference of some years. We did not get full agreement mainly in the last 5 digits in the higher coefficients and came already near to the limit of capabilities of our PC.
} 
following pairs of zeros lie on the imaginary axis $z=i y^{3}$ :

Table 1. Zeros $z_{k}= \pm \mathrm{i} y_{k}$ on the imaginary axis for the first $2 M$ approximations up to $2 M=80$.

\begin{tabular}{rlrl}
\hline $2 \mathrm{M}$ & \multicolumn{1}{c}{ zeros on imaginary axis } & $2 \mathrm{M}$ & \multicolumn{1}{c}{ zeros on imaginary axis } \\
\hline 2 & $\pm \mathrm{i} 6.57881$ & 42 & $\pm \mathrm{i} 14.1347$ \\
4 & - & 44 & $\pm \mathrm{i} 14.1347, \pm \mathrm{i} 20.2528$ \\
6 & $\pm \mathrm{i} 8.48024$ & 46 & $\pm \mathrm{i} 14.1347$ \\
8 & - & 48 & $\pm \mathrm{i} 14.1347, \pm \mathrm{i} 20.8469$ \\
10 & $\pm \mathrm{i} 10.0594$ & 50 & $\pm \mathrm{i} 14.1347, \pm \mathrm{i} 21.0934, \pm \mathrm{i} 21.8540$ \\
12 & - & 52 & $\pm \mathrm{i} 14.1347, \pm \mathrm{i} 21.0123$ \\
14 & $\pm \mathrm{i} 11.4267$ & 54 & $\pm \mathrm{i} 14.1347, \pm \mathrm{i} 21.0238, \pm \mathrm{i} 23.0702$ \\
16 & - & 56 & $\pm \mathrm{i} 14.1347, \pm \mathrm{i} 21.0218$ \\
18 & $\pm \mathrm{i} 12.6077$ & 58 & $\pm \mathrm{i} 14.1347, \pm \mathrm{i} 21.0221, \pm \mathrm{i} 24.0109$ \\
20 & - & 60 & $\pm \mathrm{i} 14.1347, \pm \mathrm{i} 21.0220$ \\
22 & $\pm \mathrm{i} 13.5513$ & 62 & $\pm \mathrm{i} 14.1347, \pm \mathrm{i} 21.0220, \pm \mathrm{i} 24.7095$ \\
24 & - & 64 & $\pm \mathrm{i} 14.1347, \pm \mathrm{i} 21.0220$ \\
26 & $\pm \mathrm{i} 14.0560$ & 66 & $\pm \mathrm{i} 14.1347, \pm \mathrm{i} 21.0220, \pm \mathrm{i} 24.9857$ \\
28 & $\pm \mathrm{i} 14.1530, \pm \mathrm{i} 15.8936$ & 68 & $\pm \mathrm{i} 14.1347, \pm \mathrm{i} 21.0220, \pm \mathrm{i} 25.0147, \pm \mathrm{i} 26.7482$ \\
30 & $\pm \mathrm{i} 14.1319$ & 70 & $\pm \mathrm{i} 14.1347, \pm \mathrm{i} 21.0220, \pm \mathrm{i} 25.0093$ \\
32 & $\pm \mathrm{i} 14.1352, \pm \mathrm{i} 17.2309$ & 72 & $\pm \mathrm{i} 14.1347, \pm \mathrm{i} 21.0220, \pm \mathrm{i} 25.0102, \pm \mathrm{i} 27.8069$ \\
34 & $\pm \mathrm{i} 14.1347$ & 74 & $\pm \mathrm{i} 14.1347, \pm \mathrm{i} 21.0220, \pm \mathrm{i} 25.0101$ \\
36 & $\pm \mathrm{i} 14.1347, \pm \mathrm{i} 18.3765$ & 76 & $\pm \mathrm{i} 14.1347, \pm \mathrm{i} 21.0220, \pm \mathrm{i} 25.0101, \pm \mathrm{i} 28.8689$ \\
38 & $\pm \mathrm{i} 14.1347$ & 78 & $\pm \mathrm{i} 14.1347, \pm \mathrm{i} 21.0220, \pm \mathrm{i} 25.0101$ \\
40 & $\pm \mathrm{i} 14.1347, \pm \mathrm{i} 19.3895$ & & $\pm \mathrm{i} 14.1347, \pm \mathrm{i} 21.0220, \pm \mathrm{i} 25.0101, \pm \mathrm{i} 30.5405$ \\
\hline & & & \\
& & &
\end{tabular}

In the following we give graphical illustrations of all zeros of the Taylor approximations in the complex $z$-plane where all zeros up to a certain order $2 M$ are taken into account and where one may see how the zeros change from an order to a higher order.

We explain first how the following figures are made. We take a certain order $2 M$ of the Xi function $\Xi(z)$ given by the truncated Taylor series (4.2) and determine numerically all of its zeros and represents them by points in the complex $(z=x+i y)$-plane where we choose the same scale on the $x^{-}$and $y$-axis. Two variants are made, first the representation by isolated points and

${ }^{3}$ From about $2 M=50$ on the values given in the third and fourth column on the right-hand side did not become fully stable in our calculations in dependence on the number of sum terms taken into account in (2.14) and the chosen upper limit of integration that is rather due to the limits of our computer capabilities. The genuine value for the third root is near to 25.0109 instead of the stabilized 25.0101 seen in the table. The fourth root is at 30.4249 . Therefore, the table reflects one such calculation. Onto the following graphical illustrations of zeros this does not have a visible influence. From the series (4.3) one may guess how difficult such calculations are even for a computer. 
second the representation by connected neighbored points. The obtained partial pictures are a little different for odd and even $M$ that is represented in Figure 4 for $M=29$ and $M=30$.

Then we calculate and represent all zeros of the Taylor approximations of $\Xi_{2 M}(z)$ (i.e. of $\left.\Xi_{2 M}(z)=0\right)$ up to a certain maximal $M$ and represent the zeros in described way by isolated points and by connected neighbored points in each of the approximations up to the maximal $M$. This is made in Figure 5 and Figure 6 for maximal $2 M=60$. These approximations capture already approximations of the first two nontrivial zeros of the Riemann zeta function on the positive $y$-axis at $y_{1} \approx 14.135$ and at $y_{2} \approx 21.022$ seen in Figure 5 by some accumulation of points at these values. Figure 6 shows the same picture with all neighbored points in each approximation joined as described. Since not all details are well recognizable in Figure 6 the same is made in Figure 7 but
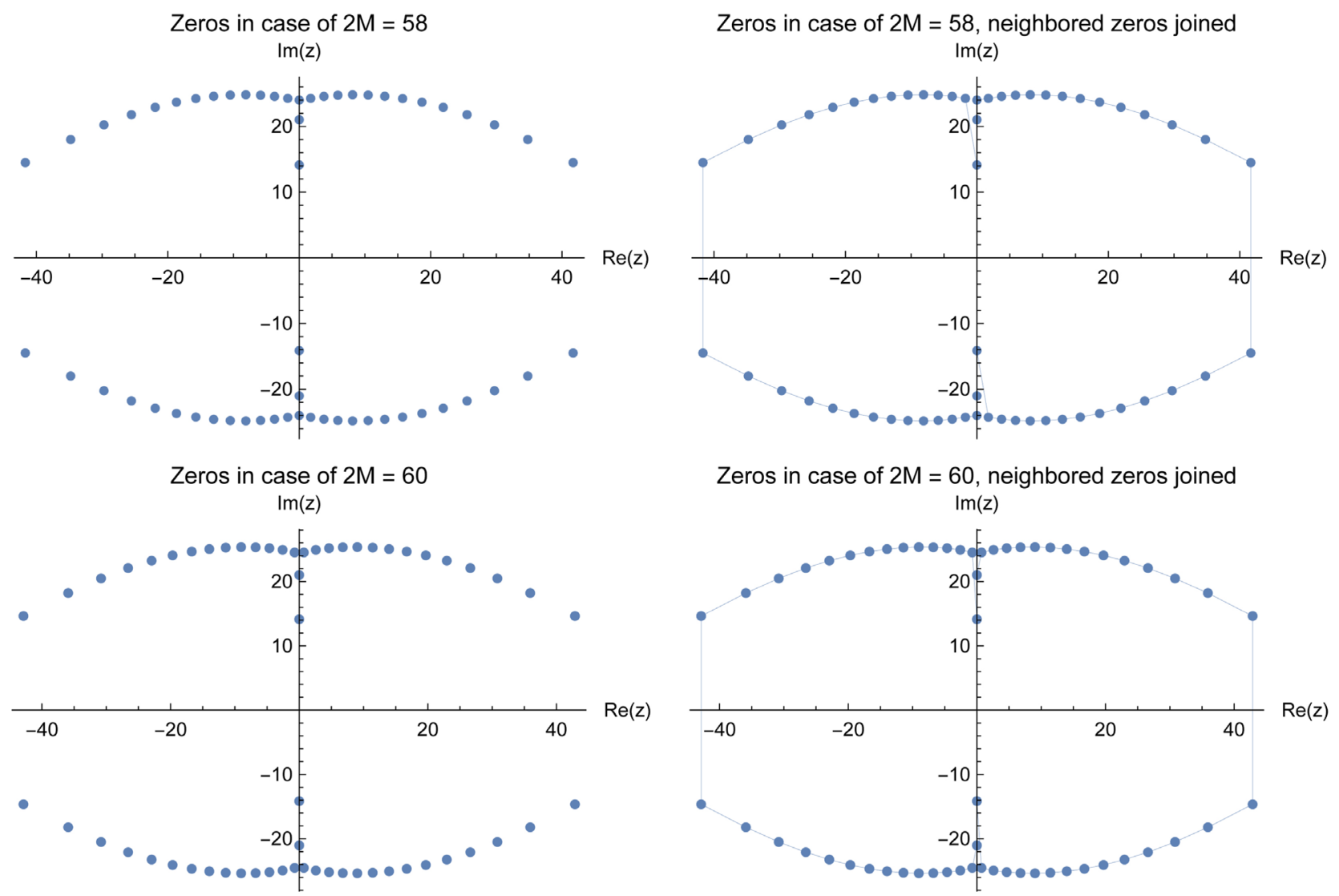

Figure 4. Zeros of $\Xi(z)=\int_{0}^{+\infty} \mathrm{d} u \Omega(u) \operatorname{ch}(u z)$ for $\Omega(u)$ according to (2.14) (i.e. for Riemann hypothesis) in approximation of the Taylor series $\sum_{m=0}^{M} \Omega_{2 m} z^{2 m}$ with $2 M=58$ and $2 M=60$. The obtained 58, respectively, 60 zeros are shown in the complex plane as points without mutual distortion of the axis lengths. In the pictures to the right-hand sides we have joined neighbored numbers. On the imaginary axes where it is not clear which zeros are neighbored to zeros off the axis we went in clockwise sense on the positive part first to the highest zero and then to the next lower zeros and so on and then from the lowest zero on the imaginary axis clockwise to the next complex zero. This shows also the way we went in the next picture where the details on the imaginary axis are not so clearly visible. The two zeros at $y_{1} \approx \pm 14.135$ and at $y_{2} \approx \pm 21.022$ on the positive and negative imaginary axes are already decoupled from the main bulk of zeros and the zeros at $y_{3} \approx \pm 25.0$ begin only to decouple. 


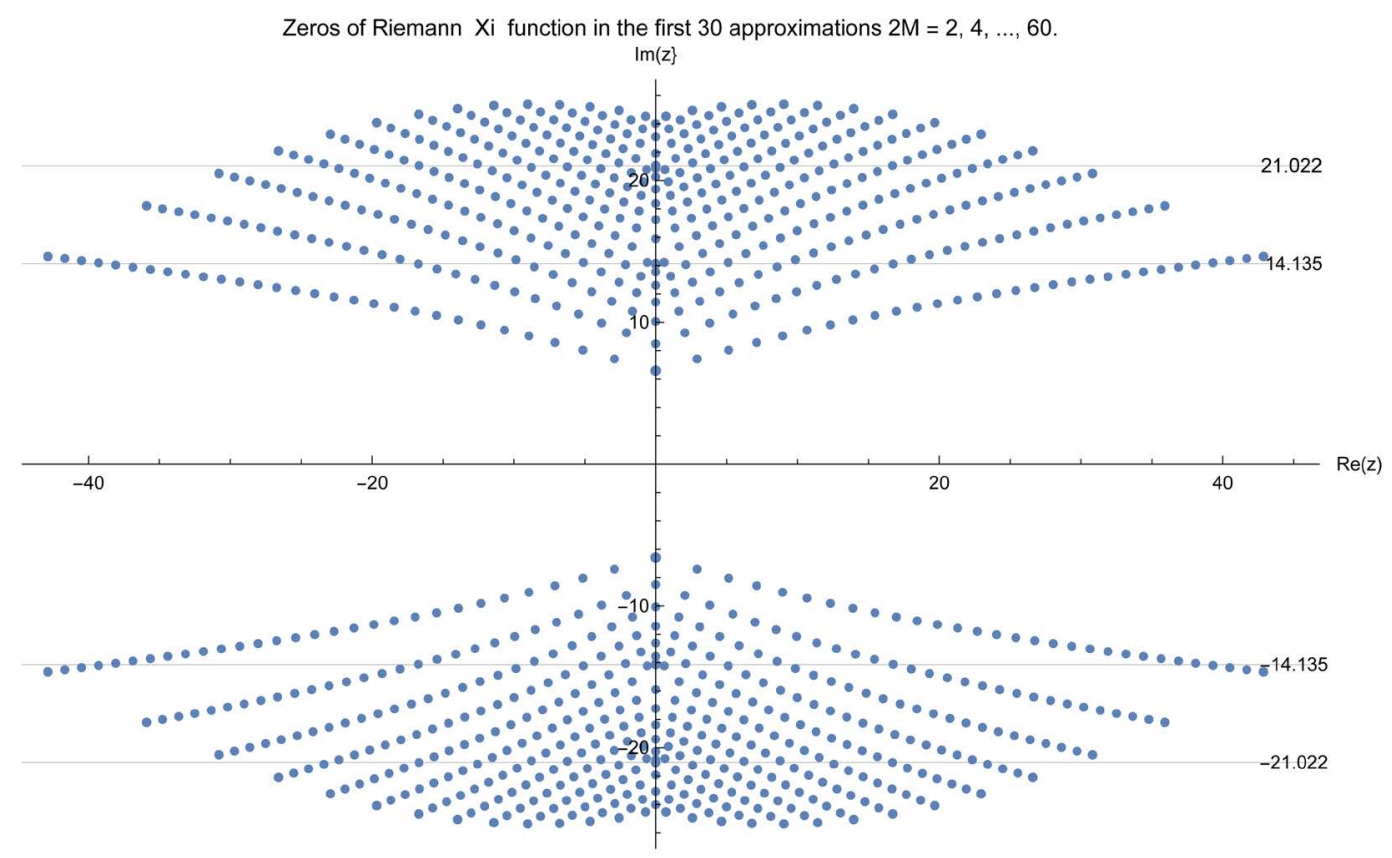

Figure 5. Zeros of $\mathrm{Xi}$ function $\Xi(z)$ to Riemann zeta function in the first 30 approximation $\Xi_{2 M}(z)=\sum_{m=0}^{M} \Omega_{2 m} z^{2 m}$ of its Taylor series with $2 M=2,4, \cdots, 60$. The neighbored zeros are not joined in each approximation. We see already the beginning accumulation of points at the first two genuine zeros of the Riemann zeta function at $y_{1} \approx \pm 14.135$ and $y_{2} \approx \pm 21.022$.

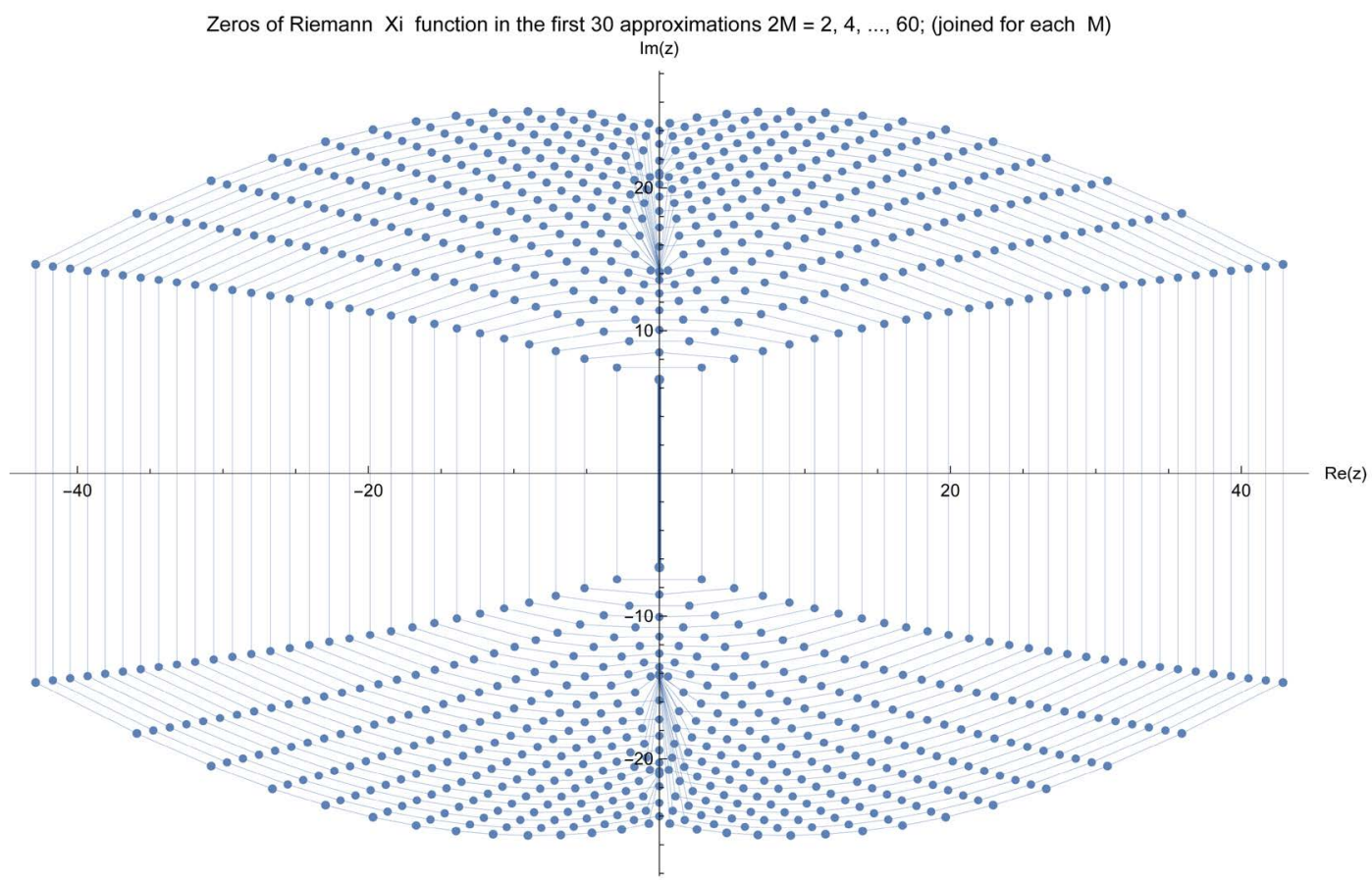

Figure 6. Zeros of $\mathrm{Xi}$ function $\Xi(z)$ to Riemann zeta function in the first 30 approximation $\Xi_{2 M}(z)=\sum_{m=0}^{M} \Omega_{2 m} z^{2 m}$ of its Taylor series with $2 M=2,4, \cdots, 60$. The neighbored zeros are joined in each approximation separately. In the immediate neighborhood of the axis the picture becomes a little confusing since then happens a big step to the smallest genuine zero of the $\mathrm{Xi}$ function and then rises up to higher zeros on the imaginary axis before it goes to the main bulk of zeros outside the imaginary axis. 


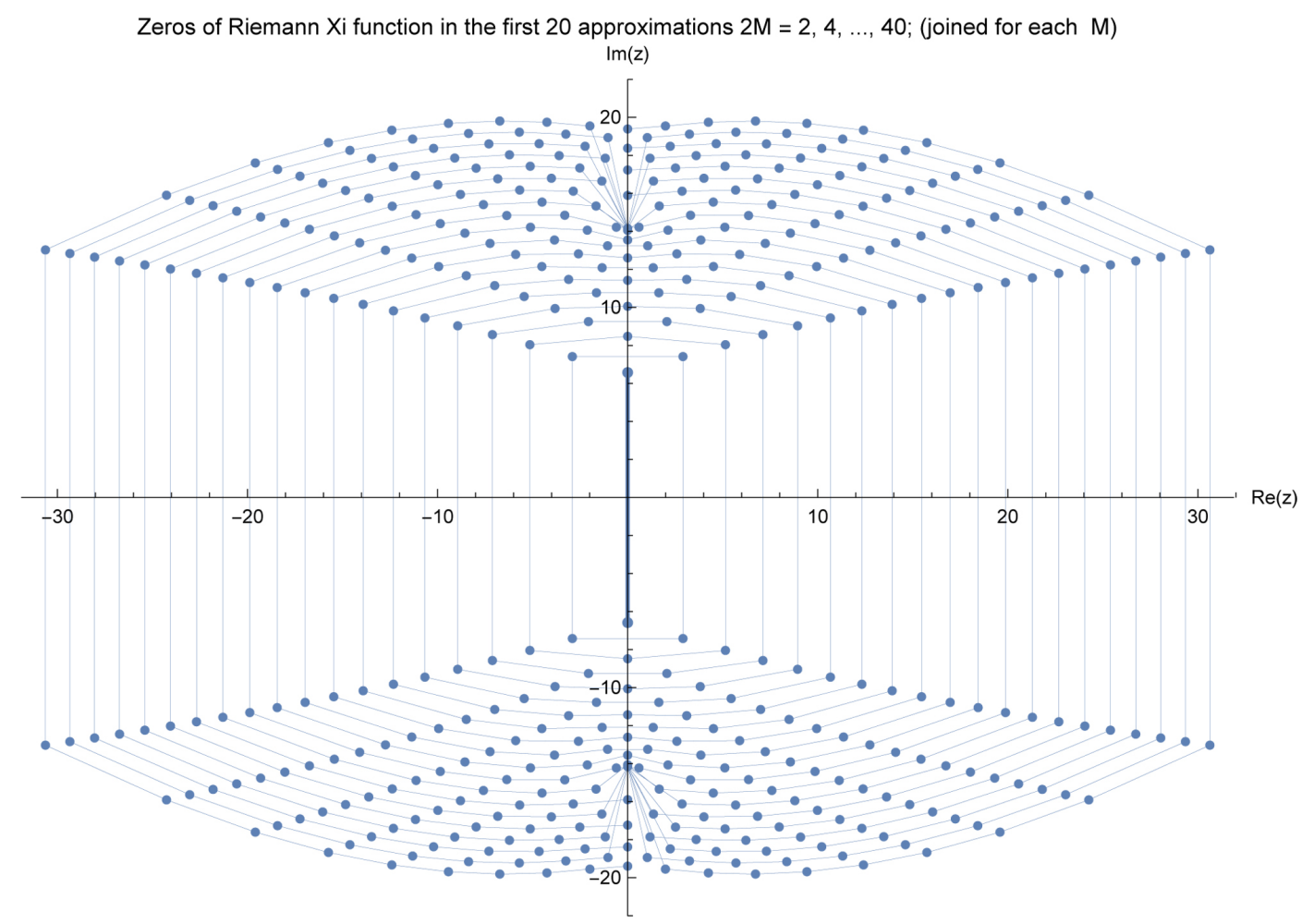

Figure 7. Zeros of $\mathrm{Xi}$ function $\Xi(z)$ to Riemann zeta function in the first 20 approximation $\Xi_{2 M}(z)=\sum_{m=0}^{M} \Omega_{2 m} z^{2 m}$ of its Taylor series with $2 M=2,4, \cdots, 40$. The neighbored zeros are joined in each approximation separately. We see that the zeros of the main bulk in the approximations go slowly but with great regularity (though not proved) to infinity for $M \rightarrow \infty$ and vanish in this way as zeros of the whole function. Only the first zero at $y_{1}= \pm 14.135$ as genuine zeros of the Riemann zeta function are seen here as decoupled and stabilized.

only for the first 40 Taylor approximations where this is clearer to see. These pictures show that the zeros on the imaginary axis stabilize from order to higher orders at the genuine zeros of the Riemann zeta function on the $y$-axis and separate themselves from the main bulk of zeros in a considered order which do not lie on the imaginary axis. That this remains in this way for $M \rightarrow \infty$ is, clearly, only a conjecture but in Section 8 we try to understand this by some analytic approximations.

In Figure 7 for the case $2 M=40$ we see only one accumulation point of the zeros on the positive and negative $y$-axis corresponding to the two zeros $y_{1} \approx \pm 14.135$. The four higher zeros on the positive $y$-axis (correspondingly negative $y$-axis) belong already to not yet stabilized approximations to the second zeros at $y_{2} \approx \pm 21.022$.

It is interesting to compare the functions $\Omega(u)$ and $\Xi(z)$ to the Riemann zeta function with a corresponding Gaussian functions $\Omega_{\mathrm{G}}(u)$ and $\Xi_{\mathrm{G}}(z)$ where the two parameters of the Gaussian function $\Omega_{\mathrm{G}}(u)$ which are the amplitude and the stretching of the parameter $u$ are chosen in the way that the first two terms of the Taylor series approximation are equal. For the function $\Omega(u)$ 


$$
\begin{aligned}
\Omega(u) & =\Omega(0)+\frac{\Omega^{(2)}(0)}{2 !} u^{2}+\frac{\Omega^{(4)}(0)}{4 !} u^{4}+\ldots \\
& \approx 1.78678760-16.7305008 u^{2}+67.6802 u^{4}-\cdots,
\end{aligned}
$$

this means for the Gaussian function $\Omega_{\mathrm{G}}(u)$

$$
\begin{aligned}
\Omega_{G}(u) & =\Omega(0) \exp \left(\frac{\Omega^{(2)}(0)}{2 \Omega(0)} u^{2}\right) \\
& =\Omega(0)+\frac{\Omega^{(2)}(0)}{2} u^{2}+\frac{\left(\Omega^{(2)}(0)\right)^{2}}{2 ! 2^{2} \Omega(0)} u^{4}+\cdots \\
& \approx 1.78678760 \cdot \exp \left(-9.363452465 u^{2}\right) \\
& \approx 1.78678760-16.7305008 u^{2}+78.3276 u^{4}-\cdots
\end{aligned}
$$

One may check that generally

$$
\Omega_{\mathrm{G}}(u) \geq \Omega(u)>0 .
$$

For the moments of these functions result the inequalities $(m=0,1,2, \cdots)$

$$
\begin{aligned}
& \Omega_{\mathrm{G}, 2 m} \equiv \frac{1}{(2 m) !} \int_{0}^{\infty} \mathrm{d} u \Omega_{\mathrm{G}}(u) u^{2 m}>\frac{1}{(2 m) !} \int_{0}^{\infty} \mathrm{d} u \Omega(u) u^{2 m}=\Omega_{2 m}>0, \\
& \Xi_{\mathrm{G}}^{(2 m)}(0) \equiv(2 m) ! \Omega_{\mathrm{G}, 2 m}>(2 m) ! \Omega_{2 m} \equiv \Xi^{(2 m)}(0) .
\end{aligned}
$$

The resulting function $\Xi_{G}(z)$ is a Gaussian function of the complex variable $z=x+\mathrm{i} y$

$$
\begin{aligned}
\Xi_{G}(z) & =\Omega(0) \sqrt{-\frac{\pi \Omega(0)}{2 \Omega^{(2)}(0)}} \exp \left(-\frac{\Omega(0)}{2 \Omega^{(2)}(0)} z^{2}\right) \\
& \approx 0.517487503 \cdot \exp \left(0.0266995535 z^{2}\right) .
\end{aligned}
$$

It possesses another amplitude in comparison to $\Xi(z)$ (i.e. to 0.497120778). Clearly, as a Gaussian function it does not possess zeros on the $y$-axis and zeros at all. The $\Omega(u)$ function to the Riemann Xi function and the considered modified Bessel functions possess the common property that they vanish in infinity more rapidly (or are even finite) than the Omega function to a Gauss function. In principle, this does not exclude Omega functions which vanish less rapidly. For example, a function $\Omega(u)=\mathrm{e}^{\alpha u},(u \geq 0, \operatorname{Re}(\alpha)>0)$ provides $\Xi(z)=\frac{\alpha}{\alpha^{2}-z^{2}}$ without zeros of $\Xi(z)$ at all in finite regions of the complex plane but with poles that regarding the zeros is the same as for a Gauss function (see Section 8 where this is explained by motion to infinity from finite approximations). However, by comparison with Gauss functions we may get inequalities for the moments of the considered Omega functions.

\section{Zeros of the Taylor Series Approximations of the Function $\frac{\operatorname{sh}(z)}{z}$}

In this Section we now come back to the modified Bessel functions $\Xi_{v}(z)$ in 
(3.7) to the Omega functions $\Omega_{v}(u)$ in (3.4). From this class of functions which in the limit $v \rightarrow \infty$ go to a Gaussian function we choose the function $\frac{\operatorname{sh}(z)}{z}$

$$
\Xi_{\frac{1}{2}}(z)=\bar{\Xi}_{\frac{1}{2}}(z)=\frac{\operatorname{sh}(z)}{z},
$$

and give graphical representations of the zeros for its Taylor series approximations

$$
\Xi_{\frac{1}{2}, 2 M}(z)=\bar{\Xi}_{\frac{1}{2}, 2 M}(z)=\sum_{m=0}^{M} \frac{z^{2 m}}{(2 m+1) !}=\sum_{m=0}^{M} \frac{\left(\frac{1}{2}\right) !}{m !\left(m+\frac{1}{2}\right) !}\left(\frac{z}{2}\right)^{2 m} .
$$

They are represented in Figure 8 and in Figure 9 up to the approximation for $2 M=60$ with the difference that in the first all zeros are presented together and in the second we have joined the neighbored zeros in each approximations. The genuine zeros of $\frac{\operatorname{sh}(z)}{z}$ lie at $z_{0}=n \pi$ according to

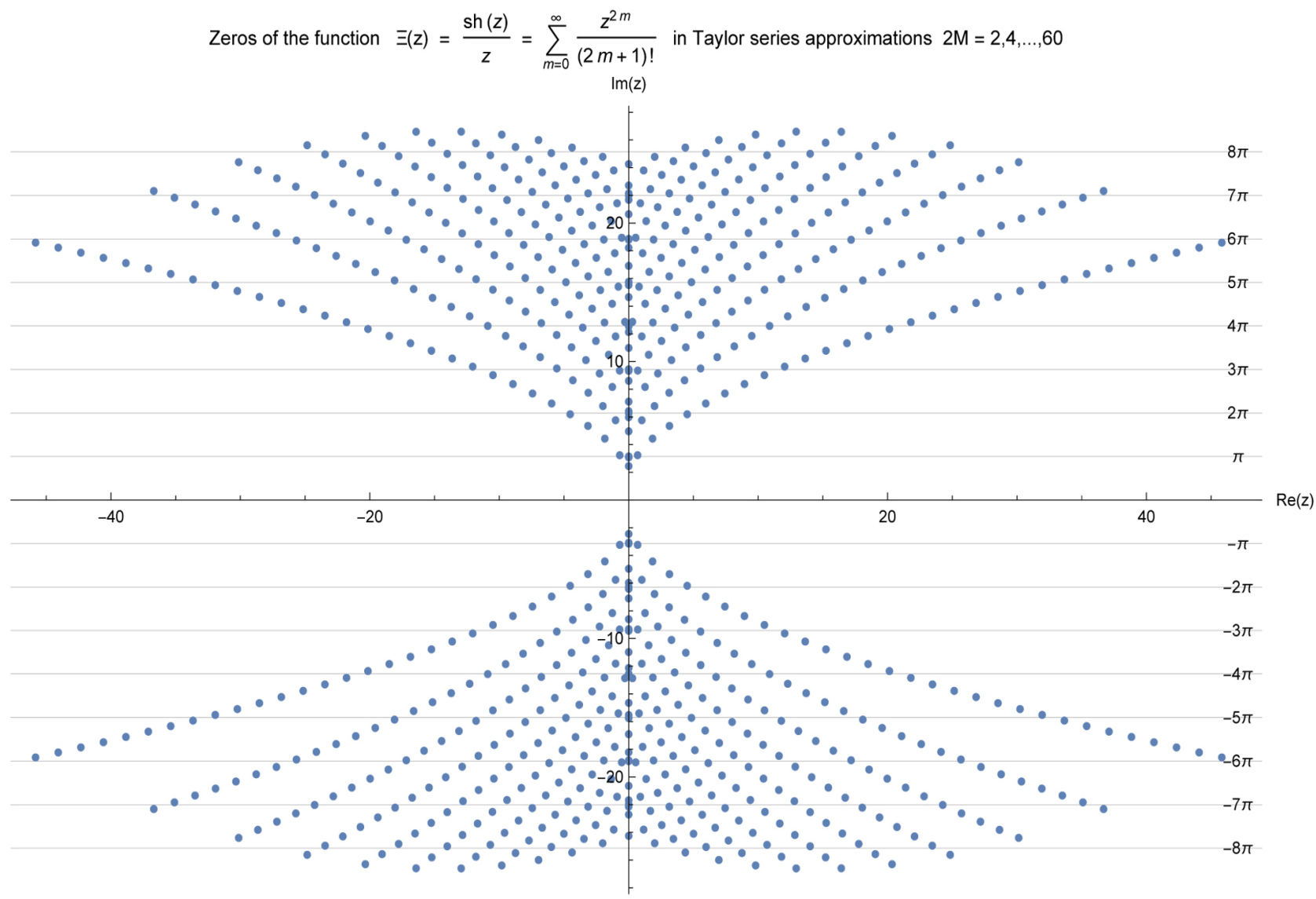

Figure 8. Zeros of the function $\Xi(z)=\frac{\operatorname{sh}(z)}{z}=\sum_{m=0}^{\infty} \frac{z^{2 m}}{(2 m+1) !}$ in the first 30 approximations $\Xi_{2 M}(z)=\sum_{m=0}^{M} \frac{z^{2 m}}{(2 m+1) !}$ of its Taylor series with $2 M=2,4, \cdots, 60$. The neighbors within an approximations are not joined and it is not fully easy to see which point belongs to a certain approximation. 


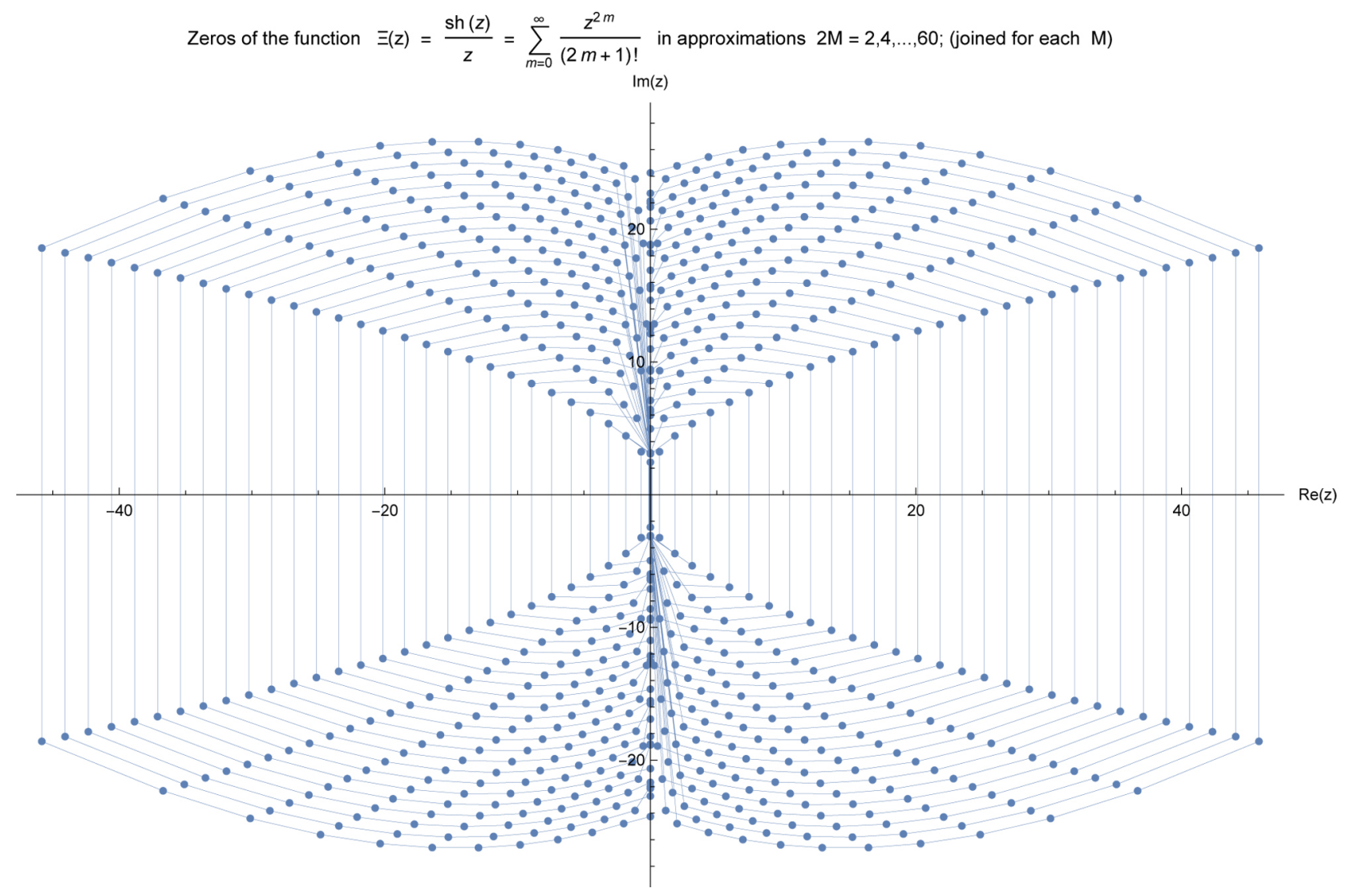

Figure 9. Zeros of the function $\Xi(z)=\frac{\operatorname{sh}(z)}{z}=\sum_{m=0}^{\infty} \frac{z^{2 m}}{(2 m+1) !}$ in the first 30 approximations $\Xi_{2 M}(z)=\sum_{m=0}^{M} \frac{z^{2 m}}{(2 m+1) !}$ of its Taylor series with $2 M=2,4, \cdots, 60$. The neighbors within each approximations are here joined. Since in each approximation a point on the positive imaginary goes approximately to the first zeros at $y_{1}= \pm \pi$ the connections on the imaginary axis are not easily recognizable.

$$
z_{0}=i n \pi,(n= \pm 1, \pm 2, \cdots) \Rightarrow \frac{\operatorname{sh}\left(z_{0}\right)}{z_{0}}=0 .
$$

We may expect that for other low values of index $v \geq 0$ in the functions $\Xi_{v}(z)$ we get similar illustrations with small topological distortions of the pictures for $\Xi_{\frac{1}{2}}(z)=\frac{\operatorname{sh}(z)}{z}$.

The function $\frac{\operatorname{sh}(z)}{z}$ possesses a peculiar importance since for monotonically decreasing functions $\Omega(u)$ (for $0 \leq u<+\infty$ with $\Omega(+\infty)=0$ ) we may apply the second mean-value theorem (Gauss-Bonnet theorem; see, e.g. Courant [14] (chap. IV), Widder [15]) to bring the integral (2.14) to the form

$$
\Xi(z)=\int_{0}^{+\infty} \mathrm{d} u \Omega(u) \operatorname{ch}(u z)=\Omega(0) \frac{\operatorname{sh}\left(w_{0}(z) z\right)}{z} .
$$

Herein, $w_{0}(z)$ is a mean value function from which we may assume that it is an analytic function of $z$ since the integral on the left-hand side depends 
analytically on $z$. In next Section we consider shortly the problem of zeros of the function $\Xi(z)$ of the form (5.4).

\section{General Conditions for Zeros of Functions $\frac{\operatorname{sh}\left(w_{0}(z) z\right)}{z}$}

The problem of zeros of functions $\Xi(z)$ of the form (5.4) leads essentially to the problem of zeros of $\operatorname{sh}\left(w_{0}(z) z\right)$ with exclusion of the zero at $z=0$. If we separate the real and imaginary part of $w_{0}(z)$ according to

$$
w_{0}(z)=u_{0}(x, y)+\mathrm{i} v_{0}(x, y), \quad z=x+i y,
$$

and if we then separate the real and imaginary part of $\operatorname{sh}\left(w_{0}(z) z\right)$ the function (5.4) can be written in the form (the separation of real and imaginary part in the first factor is uninteresting since a zero at $z=0$ is excluded)

$$
\begin{aligned}
\Xi(x+\mathrm{i} y)= & \frac{\Omega(0)}{x+\mathrm{i} y} \operatorname{sh}\left(u_{0}(x, y) x-v_{0}(x, y) y+\mathrm{i}\left(u_{0}(x, y) y+v_{0}(x, y) x\right)\right) \\
= & \frac{\Omega(0)}{x+\mathrm{i} y}\left\{\operatorname{sh}\left(u_{0}(x, y) x-v_{0}(x, y) y\right) \cos \left(u_{0}(x, y) y+v_{0}(x, y) x\right)\right. \\
& \left.+\mathrm{ich}\left(u_{0}(x, y) x-v_{0}(x, y) y\right) \sin \left(u_{0}(x, y) y+v_{0}(x, y) x\right)\right\} .
\end{aligned}
$$

From both forms of the right-hand side in (6.2) we find that for zeros of $\Xi(x+\mathrm{i} y)$ the following two conditions [11]

$$
\begin{gathered}
u_{0}(x, y) y+v_{0}(x, y) x=n \pi, \quad n= \pm 1, \pm 2, \cdots, \\
u_{0}(x, y) x-v_{0}(x, y) y=0,
\end{gathered}
$$

have to be satisfied at the same time and this is necessary and sufficient.

We now consider the special case of $\Xi(z)$ on the imaginary axis $y$ and find from (6.2)

$$
\Xi(\mathrm{i} y)=\frac{\Omega(0)}{y} \sin \left(\left(u_{0}(0, y)+\mathrm{i} v_{0}(0, y)\right) y\right)=\Omega(0) \frac{\sin \left(w_{0}(y) y\right)}{y} .
$$

Since due to symmetries (2.8) the function $\Xi(i y)$ has to be a real-valued function that for $y \neq 0$ in (6.4) is only possible if $v_{0}(0, y)$ vanishes we find on the imaginary axis

$$
v_{0}(0, y)=0 \Rightarrow \Xi(\mathrm{i} y)=\frac{\Omega(0)}{y} \sin \left(u_{0}(0, y) y\right)=\int_{0}^{+\infty} \mathrm{d} u \Omega(u) \cos (u y) .
$$

This follows also immediately by application of the second mean-value theorem to the integral for $\Xi$ (iy) where the mean value can only take on real values here in dependence on $y$ as parameter. Thus for zeros on the imaginary axis $(x=0)$ the two conditions (6.3) reduce to only one condition

$$
u_{0}(0, y) y=n \pi, \quad n= \pm 1, \pm 2, \cdots .
$$

The condition in the second line of (6.3) is then identically satisfied. If one knows the function $u_{0}(0, y)$ one may determine the zeros on the imaginary 
axis analytically or, at least, numerically but for many problems including the considered one it is not necessary to know these zeros explicitly.

We return to the general case of general values $x$ on the real axis with the conditions (6.3) for zeros. From (2.8) follows that $\Xi(z)$ should be an analytic function for all $z$ for which the integral exists that means to satisfy the CauchyRiemann equations. For a general analytic function $w(z)=w(x+\mathrm{i} y)=u(x, y)+\mathrm{i} v(x, y)$ one can derive by integration of the Cauchy-Riemann equations the following relations in case of $v(0, y)=0$ [11]

$$
u(x, y)=\cos \left(x \frac{\partial}{\partial y}\right) u(0, y), \quad v(x, y)=-\sin \left(x \frac{\partial}{\partial y}\right) u(0, y) .
$$

Now come into play the following operator identities (operator identities are such identities which can be applied to arbitrary functions to provide function identities) [11]

$$
\begin{aligned}
& \cos \left(x \frac{\partial}{\partial y}\right) y=y \cos \left(x \frac{\partial}{\partial y}\right)-x \sin \left(x \frac{\partial}{\partial y}\right), \\
& \sin \left(x \frac{\partial}{\partial y}\right) y=x \cos \left(x \frac{\partial}{\partial y}\right)+y \sin \left(x \frac{\partial}{\partial y}\right) .
\end{aligned}
$$

More general identities of such kind can be derived by representing the Cosine and Sine functions by Exponential functions and using that $\exp \left( \pm \mathrm{i} x \frac{\partial}{\partial y}\right)$ applied to analytic functions $f(y)$ displace the argument of these functions to $f(y \pm \mathrm{ix})$ that is discussed in [11]. Using (6.7) and (6.8) we may write the conditions for zeros (6.3) in the following way

$$
\begin{aligned}
& n \pi=\left\{y \cos \left(x \frac{\partial}{\partial y}\right)-x \sin \left(x \frac{\partial}{\partial y}\right)\right\} u_{0}(0, u)=\cos \left(x \frac{\partial}{\partial y}\right) y u_{0}(0, y), \\
& 0=\left\{x \cos \left(x \frac{\partial}{\partial y}\right)+y \sin \left(x \frac{\partial}{\partial y}\right)\right\} u_{0}(0, y)=\sin \left(x \frac{\partial}{\partial y}\right) y u_{0}(0, y) .
\end{aligned}
$$

If we now apply the operator $\cos \left(x \frac{\partial}{\partial y}\right)$ to the first of these conditions and the operator $\sin \left(x \frac{\partial}{\partial y}\right)$ to the second one we obtain

$$
\cos ^{2}\left(x \frac{\partial}{\partial y}\right) y u_{0}(0, y)=n \pi, \quad \sin ^{2}\left(x \frac{\partial}{\partial y}\right) y u_{0}(0, y)=0 .
$$

By addition of both equalities using the operator identity

$$
\cos ^{2}\left(x \frac{\partial}{\partial y}\right)+\sin ^{2}\left(x \frac{\partial}{\partial y}\right)=1
$$

follows from (6.10)

$$
y u_{0}(0, y)=n \pi, \quad(n= \pm 1, \pm 2, \cdots) .
$$

This consequence for zeros results from both conditions (6.3) and provides a 
necessary condition for the imaginary values $y$ of all zeros for which their imaginary value has to agree with one of the solutions for zeros on the imaginary axis. Thus we have to take all solutions $y$ for zeros on the imaginary axis which we denote now by $y_{0}$ and which satisfy the conditions

$$
y_{0} u_{0}\left(0, y_{0}\right)=n \pi \Leftrightarrow \frac{\sin \left(y_{0} u_{0}\left(0, y_{0}\right)\right)}{y_{0}}=0,
$$

and have to look for solutions $x$ at the same time of both equations

$$
\begin{aligned}
& u_{0}\left(x, y_{0}\right) y_{0}+v\left(x, y_{0}\right) x=n \pi, \\
& u_{0}\left(x, y_{0}\right) x-v\left(x, y_{0}\right) y_{0}=0 .
\end{aligned}
$$

If one finds such solutions $x=x_{0}$ of one of the conditions then it is almost in all cases not a solution of the other condition and, therefore, $x_{0} \pm \mathrm{i} y_{0}$ is then not a zero. Only in very few cases of $y_{0}$ depending on the function $\Omega(u)$ in (2.8) one would expect to find values $x=x_{0} \neq 0$ as solution of both conditions (6.14). This is a very strong restriction to the satisfaction of both conditions (6.14). It seems that the set of functions $\Omega(u)$ with such a property can be only a set of measure zero within the set of all possible functions $\Omega(u)$ in (2.8). One such case is when $\Omega(u)$ is stepwise constant and monotonically decreasing with equal lengths $u_{0}$ of the steps and this is the only case (Appendix A). The corresponding functions $\Xi(z)$ are then superpositions of functions $\frac{\operatorname{sh}\left(m u_{0} z\right)}{z},(m=1,2, \cdots)$ with different amplitudes. This was not correctly discussed in [11] and Katsnelson [16] showed an error in a short Email ${ }^{4}$ and we recognized it [17] but it was already seen in [11] that the possible zeros off the imaginary axis, i.e. $z_{0}=x_{0} \pm \mathrm{i} y_{0}$ of $\Xi(z)$ with $x_{0} \neq 0$, must possess imaginary parts $y_{0}$ which agree with one of the zeros on the imaginary axis. Reactions regarding concern about the applicability of the Bonnet theorem for present case were expressed by others, in particular, in a nice Email by Gélinas [18] with appended file but I could not find it published now. This also means that the Riemann hypothesis which is the absence of zeros of the Riemann Xi function off the imaginary axis through $x=\frac{1}{2}$ was not correctly solved by the second mean-value approach to this time although it was very improbable that a nontrivial zero of the Riemann zeta function off the imaginary axis has exactly the same imaginary value as that of one on the imaginary axis through $x=\frac{1}{2}$. We add now in Appendix A a further important building stone to a full proof of the Riemann hypothesis by the second mean-value theorem which seems to be

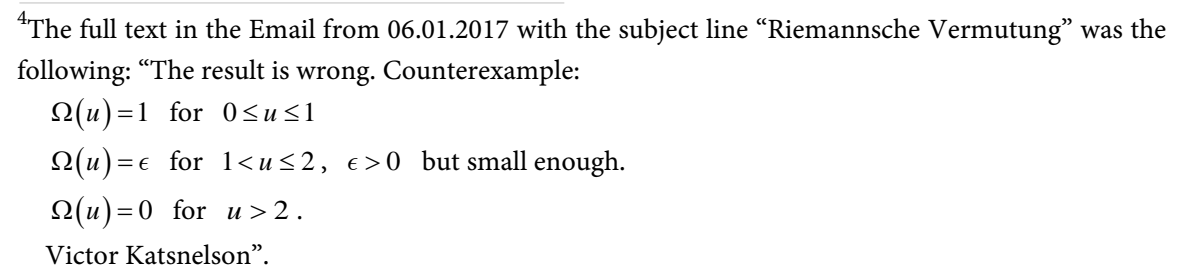


"deciding" (?).

\section{Zeros of Taylor Series Approximations of Gauss Function and Absence of Genuine Zeros Understood in Uncommon Way}

We now consider the Gauss function $\Xi_{G}(z)=\exp \left(z^{2}\right)$ and calculate all zeros of its low-order Taylor series approximations $\Xi_{\mathrm{G}, 2 M}(z)$

$$
\Xi_{\mathrm{G}, 2 M}(z)=\sum_{m=0}^{M} \frac{z^{2 m}}{m !}, \quad(M=1,2, \cdots) .
$$

In Figure 10 and Figure 11 we illustrate then their zeros up to a certain maximal $M$ in the same way as we did this for the Xi function to the Riemann zeta function in Section 4 and for the Xi functions to the modified Bessel function $\frac{\operatorname{sh}(z)}{z}$ in Section 5. This provides good analogies and shows the

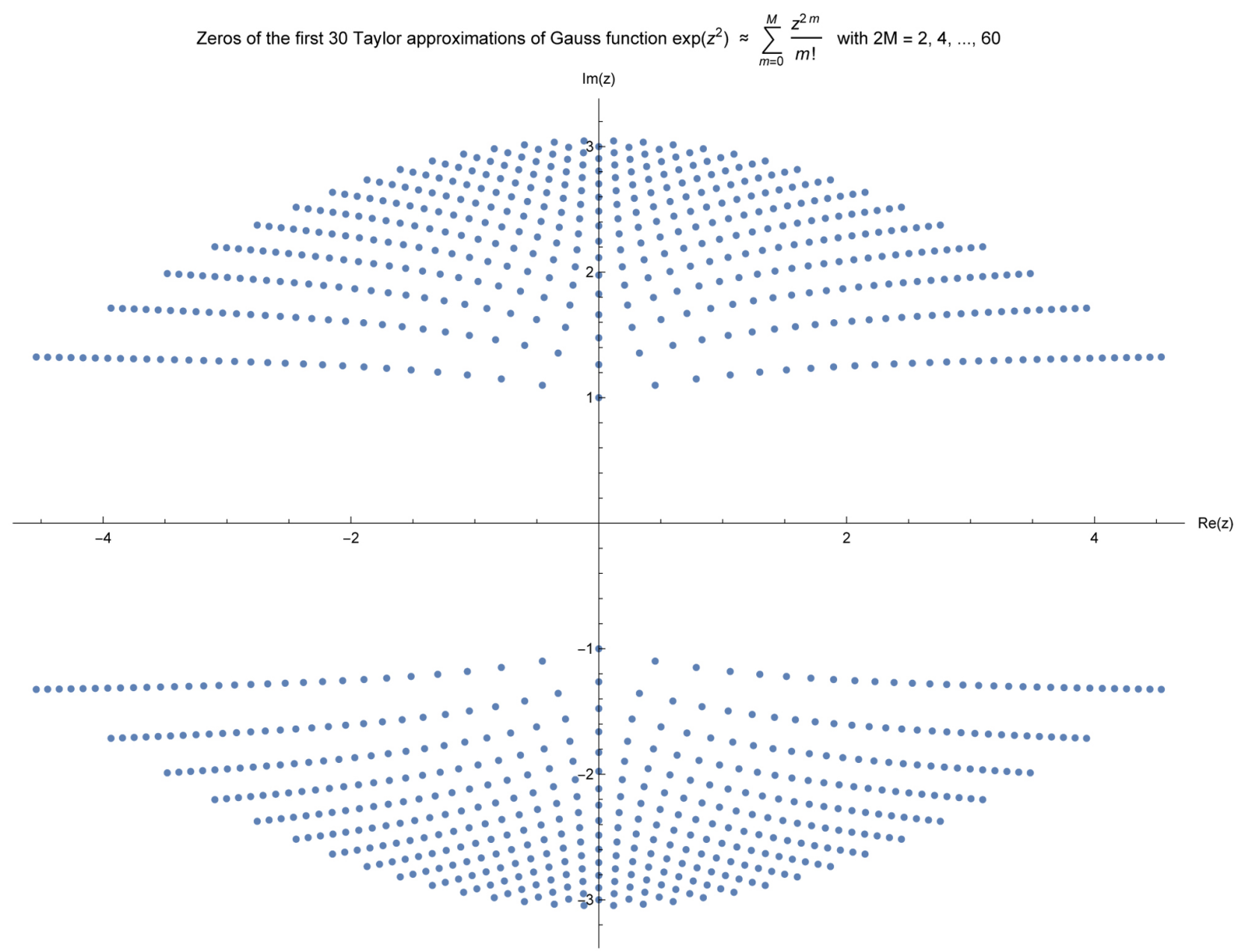

Figure 10. Zeros of Gaussian Function $\Xi(z)=\exp \left(z^{2}\right)$ in the first 30 approximations $\Xi_{2 M}(z)=\sum_{m=0}^{M} \frac{z^{2 m}}{m !}$ of its Taylor series with $2 M=2,4, \cdots, 60$. The neighbors within an approximations are here not joined and it is not easily to see which point belongs to a certain approximation. 


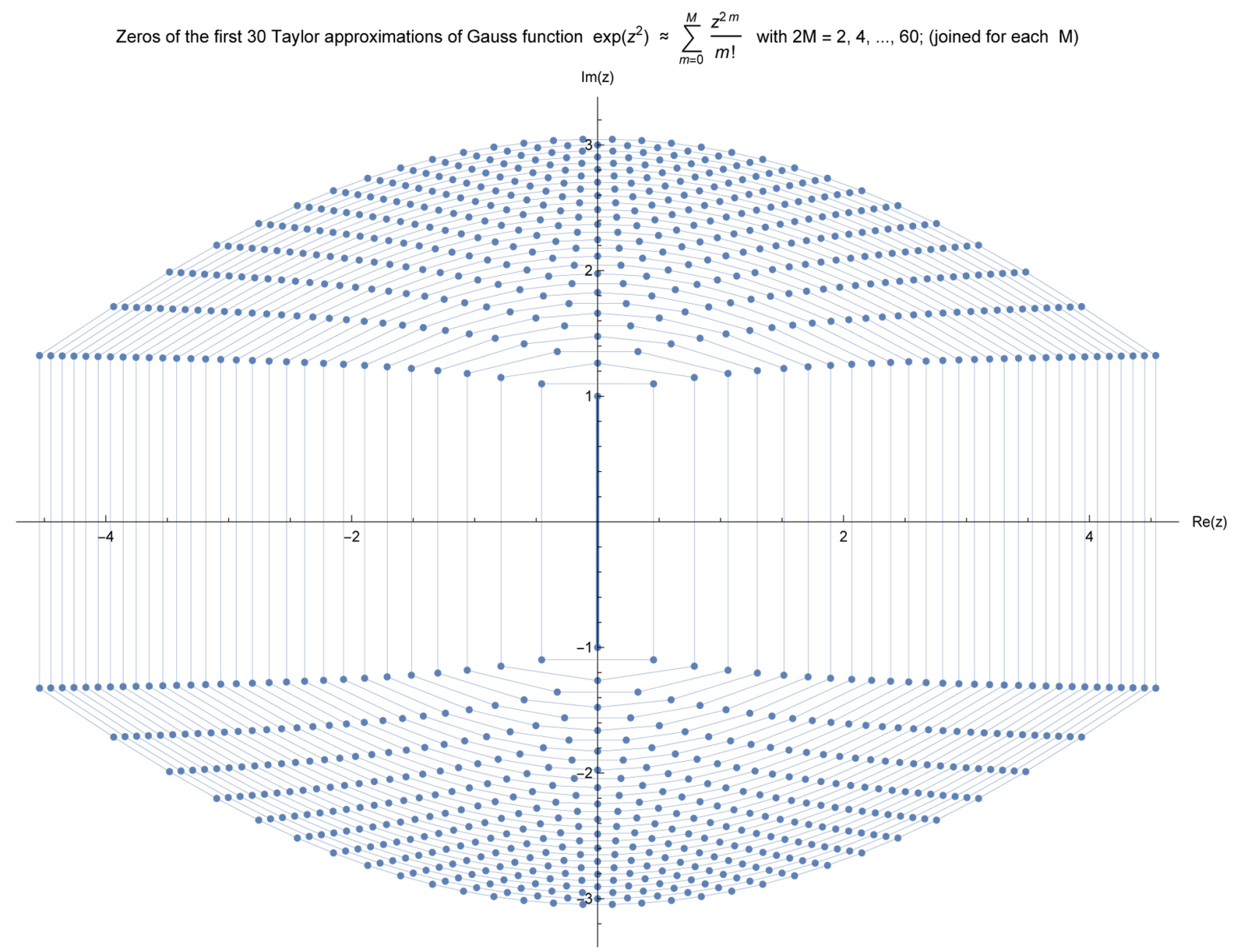

Figure 11. Zeros of Gaussian Function $\Xi(z)=\exp \left(z^{2}\right)$ in the first 30 approximations $\Xi_{2 M}(z)=\sum_{m=0}^{M} \frac{z^{2 m}}{m !}$ of its Taylor series with $2 M=2,4, \cdots, 60$. The neighbors within an approximations are joined in each approximation. The whole bulk of zeros in each approximation goes here with increasing higher approximation to infinity.

essential differences in the pictures for the Gauss function to the other discussed functions with genuine zeros when we increase the order of approximation. It becomes obvious that in the limiting transition to the Gaussian function $\exp \left(z^{2}\right)$ all zeros of the finite-order Taylor approximations go to infinity with great uniformity from order to higher order and the Gaussian function does not possess genuine zeros in finite regions of the complex $z$-plane. In Section 3 we established in (3.13) a limiting transition from modified Bessel functions with stretched arguments to a Gauss function and it was interesting to see how the zeros of finite-order Taylor series approximations move from approximation to the next higher approximation in the limiting case to infinity. The pictures for the Gaussian functions are very homogenous in contrast to the considered functions with genuine zeros. This provides an uncommon view onto the absence of zeros of Gaussian functions $\exp \left(\frac{a^{2} z^{2}}{4}\right)$ despite a representation in 
the form (2.7).

\section{Approximations of the Zeros from One to the Next Higher Orders}

The zeros in each approximation for the considered functions are either pairs $\left(z_{k}\right) \equiv\left(\mathrm{i} y_{k},-\mathrm{i} y_{k}\right)$ on the imaginary axis or in majority quadruples $\left(z_{k}\right) \equiv\left(x_{k}+\mathrm{i} y_{k}, x_{k}-\mathrm{i} y_{k},-x_{k}+\mathrm{i} y_{k},-x_{k}-\mathrm{i} y_{k}\right)$ and there was no doubt which are pairs and which are quadruples even in case that their real part $x_{k}$ is small compared with maximal modulus of the zeros and since the whole number of zeros has to be $2 M$. It is noticeable that new zeros on the imaginary axis when they first appear in the $2 M$-th approximation may disappear in the next higher $(2 M+2)$-th approximation from the imaginary axis and reappear then in the $(2 M+4)$-th approximation as can be also seen from Table 1 . In every case when there appeared a new zero on the imaginary axis the next lower zeros began to stabilize and to decouple from the main bulk of zeros in the complex domain and stabilize there in each new $(2 M+2)$-th approximation as we may see in Figures 4-7 for the Xi function $\Xi(z)$ to the Riemann hypothesis and in Figure 8 and Figure 9 for a modified Bessel function $\frac{\operatorname{sh}(z)}{z}$. In Figure 10 and Figure 11 for the function $\exp \left(z^{2}\right)$ the zeros on the imaginary axis show a similar picture with alternatingly generating and not generating zeros on the imaginary axis from one approximation $2 M$ to the next higher approximation $2 M+2$. The main bulk of zeros in the complex domain in all these pictures drifts with their modulus to infinity although very slowly that we can see in Figure 11. In the other pictures this is the main bulk of zeros which does not correspond to genuine zeros of the considered functions whereas the lower zeros on the imaginary axis stabilized more and more to the genuine zeros.

To understand the discussed behavior of the zeros from order to next higher order we try to discuss this now in some approximation. We suppose that we have the Taylor series approximation of a Xi function $\Xi(z)$ in the order $2 M$ in the form

$$
\Xi_{2 M}(z)=\sum_{m=0}^{M} \Omega_{2 m} z^{2 m} .
$$

We assume that $z_{0}$ is an exact zero in $2 M$-th approximation that means a solution of the equation

$$
\sum_{m=0}^{M} \Omega_{2 m} z_{0}^{2 m}=0
$$

Then we try to calculate next higher solutions $z=z_{0}+\Delta z$ which are near to $z_{0}$ from the next higher $2 M+2$ approximation which satisfies the equation

$$
\sum_{m=0}^{M} \Omega_{2 m}\left(z_{0}+\Delta z\right)^{2 m}+\Omega_{2 M+2}\left(z_{0}+\Delta z\right)^{2 M+2}=0
$$

In full generality this equation would provide the $2 M+2$ solutions of zeros from only one arbitrary solution $z_{0}$ in the considered approximation but in 
such generality we cannot and do not want to solve it. Well soluble is the equation for additions to $z_{0}$ leading to a quadratic equation for $\Delta z$. This provides two solutions in a neighborhood for the considered $z_{0}$. Thus we now make an expansion of the left-hand side in (8.3) up to quadratic terms in $\Delta z$

$$
\begin{aligned}
0= & \underbrace{\sum_{m=0}^{M} \Omega_{2 m} z_{0}^{2 m}}_{=0}+\sum_{m=0}^{M} 2 m \Omega_{2 m} z_{0}^{2 m-1} \Delta z+\sum_{m=0}^{M} m(2 m-1) \Omega_{2 m} z_{0}^{2 m-2}(\Delta z)^{2} \\
& +\Omega_{2 M+2}\left(z_{0}^{2 M+2}+(2 M+2) z_{0}^{2 M+1} \Delta z+(M+1) 2 M z_{0}^{2 M}(\Delta z)^{2}\right) .
\end{aligned}
$$

If we neglect from the additional terms of the $(2 M+2)$-th approximation the terms proportional to $\Delta z$ and $(\Delta z)^{2}$ as small terms we obtain the following quadratic equation for $\frac{\Delta z}{z_{0}}$

$$
\left(\frac{\Delta z}{z_{0}}\right)^{2}+\frac{\sum_{m=0}^{M} 2 m \Omega_{2 m} z_{0}^{2 m}}{\sum_{m=0}^{M} m(2 m-1) \Omega_{2 m} z_{0}^{2 m}} \frac{\Delta z}{z_{0}}+\frac{\Omega_{2 M+2} z_{0}^{2 M+2}}{\sum_{m=0}^{M} m(2 m-1) \Omega_{2 m} z_{0}^{2 m}}=0 .
$$

The two solutions of this equation are

$$
\begin{aligned}
\frac{\Delta z}{z_{0}}= & \frac{1}{2}\left\{-\frac{\sum_{m=0}^{M} 2 m \Omega_{2 m} z_{0}^{2 m}}{\sum_{m=0}^{M} m(2 m-1) \Omega_{2 m} z_{0}^{2 m}}\right. \\
& \pm \sqrt{\left.\left(\frac{\left.\sum_{m=0}^{M} 2 m \Omega_{2 m} z_{0}^{2 m}\right)^{2}}{\sum_{m=0}^{M} m(2 m-1) \Omega_{2 m} z_{0}^{2 m}}\right)^{2}-\frac{4 \Omega_{2 M+2} z_{0}^{2 M+2}}{\sum_{m=0}^{M} m(2 m-1) \Omega_{2 m} z_{0}^{2 m}}\right\},},
\end{aligned}
$$

or more compactly written

$$
\frac{\Delta z}{z_{0}}=-\frac{\Xi_{2 M}^{(1)}\left(z_{0}\right)}{z_{0} \Xi_{2 M}^{(2)}\left(z_{0}\right)} \pm \sqrt{\left(\frac{\Xi_{2 M}^{(1)}\left(z_{0}\right)}{z_{0} \Xi_{2 M}^{(2)}\left(z_{0}\right)}\right)^{2}-\frac{2 \Omega_{2 M+2} z_{0}^{2 M}}{\Xi_{2 M}^{(2)}\left(z_{0}\right)}} .
$$

where $\Xi_{2 M}^{(n)}\left(z_{0}\right)$ are the $n$-th derivatives of $\Xi_{2 M}^{(n)}(z)$ taken at $z=z_{0}$. One may express this also by the logarithmic derivative of $\Xi_{2 M}^{(1)}(z)$ that, however, is inconvenient since it goes into the formula as a denominator.

For the most interesting case of points $z_{0}=\mathrm{i} y_{0}$ on the imaginary axis we find from (8.6)

$$
\begin{aligned}
\frac{\Delta z}{y_{0}}= & \frac{i}{2}\left\{-\frac{\sum_{m=0}^{M}(-1)^{m} 2 m \Omega_{2 m} y_{0}^{2 m}}{\sum_{m=0}^{M}(-1)^{m} m(2 m-1) \Omega_{2 m} y_{0}^{2 m}}\right. \\
& \left. \pm \sqrt{\left(\frac{\sum_{m=0}^{M}(-1)^{m} 2 m \Omega_{2 m} y_{0}^{2 m}}{\sum_{m=0}^{M}(-1)^{m} m\left((2 m-1) \Omega_{2 m} y_{0}^{2 m}\right.}\right)^{2}-\frac{4(-1)^{M+1} \Omega_{2 M+2} y_{0}^{2 M+2}}{\sum_{m=0}^{M}(-1)^{m} m(2 m-1) \Omega_{2 m} y_{0}^{2 m}}}\right\} .
\end{aligned}
$$


The sum terms in braces are real ones but likely change their signs at the zeros (we think that it can be proved). The first sum term in braces changes a little the imaginary value of the root but the second term with the root with the two possible signs must be real or imaginary. In case of negative values of the content of the root it gives two imaginary values and together with the whole expression it provides two corrections off the imaginary axis. This can be seen in many of the picture for the roots. However a full discussion of the behavior from approximation to next higher approximation by (8.8) is complicated and has to describe how the roots stabilize on the imaginary axis in dependence on the moments. Such a discussion we cannot give to this time.

\section{About the Zeros of an Unorthodox Function in Their Taylor Series Approximations}

We consider here shortly for comparison with the pictures for the up to now discussed functions with a representation of the principal form (2.8) the following unorthodox entire function of an essential other kind

$$
f(z)=\sum_{n=0}^{\infty} \frac{z^{n}}{\sqrt{n !}}
$$

with respect to the zeros in its finite Taylor series approximations. This function plays a role for the calculation of the properties of coherent phase states [19].

The function $f(z)$ in (9.1) possesses even and odd powers of variable $z$ and, therefore it is not symmetrical with mirror symmetry to the real and imaginary axis but only symmetrical to the real axis. Taking separately the even and odd powers of $z$ and applying the duplication formula for the factorials one may represent (9.1) in the form

$$
f(z)=\pi^{\frac{1}{4}} \sum_{m=0}^{\infty} \frac{z^{2 m}}{2^{m} m !}\left(\sqrt{\frac{m !}{\left(m-\frac{1}{2}\right)}}+\frac{z}{\sqrt{2}} \sqrt{\frac{m !}{\left(m+\frac{1}{2}\right)}}\right), \quad f(0)=1,
$$

with the possible approximations in the coefficients for $m \gg 1$ (see (3.11))

$$
\sqrt{\frac{m !}{\left(m-\frac{1}{2}\right) !}} \approx\left(m+\frac{1}{4}\right)^{\frac{1}{4}}, \sqrt{\frac{m !}{\left(m+\frac{1}{2}\right) !}} \approx\left(m+\frac{3}{4}\right)^{-\frac{1}{4}} .
$$

This shows that $f(z)$ for real positive $z=x>0$ grows moderately faster than the function $\exp \left(\frac{x^{2}}{2}\right)$. Therefore, the function $\exp \left(-\frac{z^{2}}{2}\right) f(z)$ is moderately increasing for real $z=x>0$ and moderately decreasing for $z=x<0 \quad$ with the Taylor series

$$
\exp \left(-\frac{z^{2}}{2}\right) f(z)=\pi^{\frac{1}{4}} \sum_{l=0}^{\infty} \frac{z^{2 l}}{2^{l} l !} \sum_{m=0}^{l} \frac{(-1)^{l-m} l !}{m !(l-m) !}\left(\sqrt{\frac{m !}{\left(m-\frac{1}{2}\right)}}+\frac{z}{\sqrt{2}} \sqrt{\frac{m !}{\left(m+\frac{1}{2}\right)}}\right),
$$


and, clearly, possesses the same zeros as the function $f(z)$.

We now give a graphical representation of the zeros of the Taylor series approximations

$$
f_{N}(z)=\sum_{n=0}^{N} \frac{z^{n}}{\sqrt{n !}}
$$

of the function $f(z)$ up to $N=120$ in Figure 12. Apparently, the computer calculated correctly up to this high approximation that we judged only from the optical impression of the figure in comparison to figures of such kind for smaller values $N$. To join neighbored points of each Taylor series approximation, at least, for such high maximal $N$ becomes unfavorable.

In Figure 12 we see the first three pairs of zeros as some accumulation points.

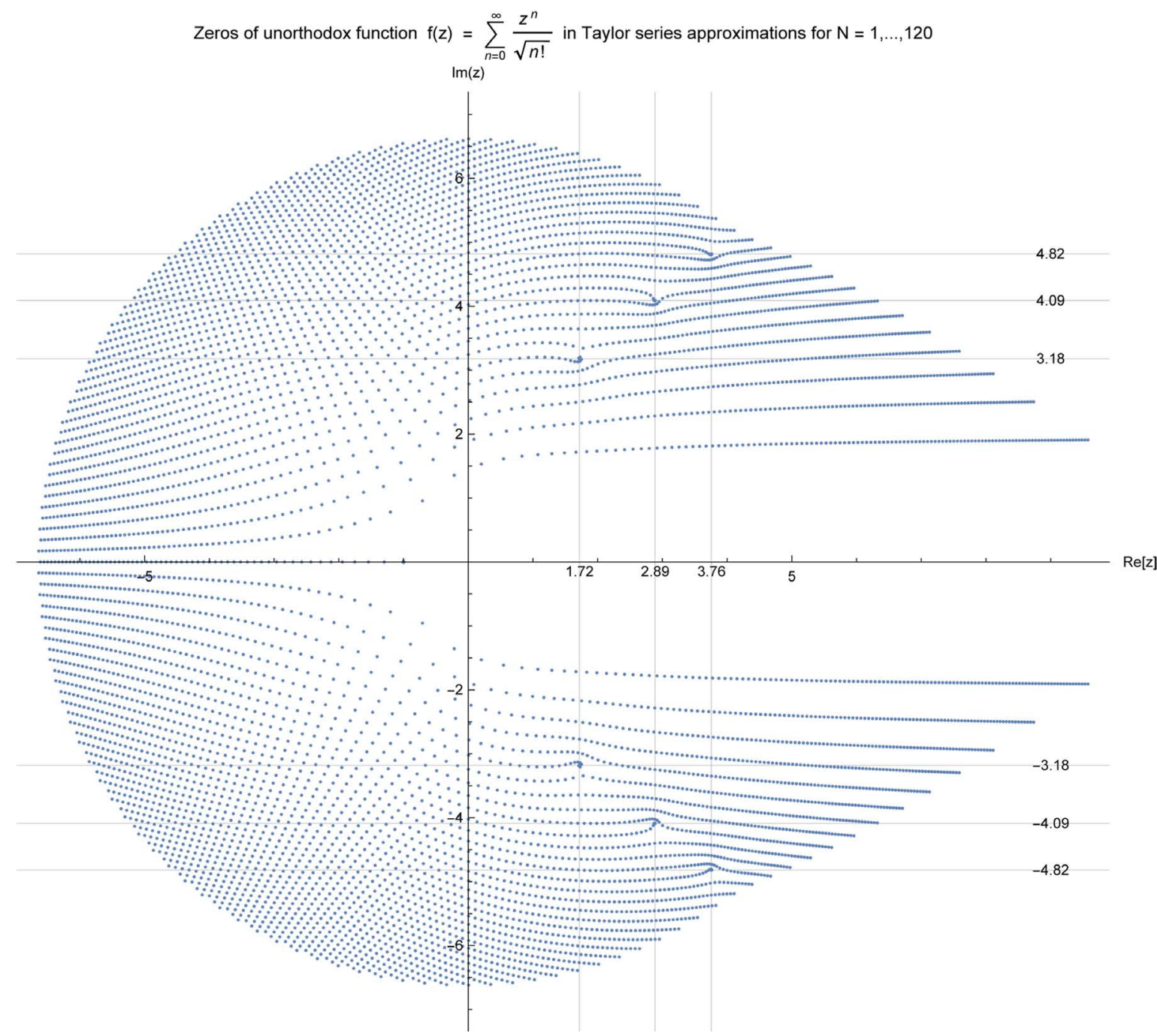

Figure 12. Zeros of unorthodox though entire function $f(z)=\sum_{n=0}^{\infty} \frac{z^{n}}{\sqrt{n !}}$ in its first 120 Taylor approximations $f_{N}(z)=\sum_{n=0}^{N} \frac{z^{n}}{\sqrt{n !}}$ with $N=1,2, \cdots, 120$. The first pairs of zeros at $z_{1}=1.71697 \pm \mathrm{i} 3.18011, \quad z_{2}=2.88669 \pm \mathrm{i} 4.09032$, $Z_{3}=3.75716 \pm \mathrm{i} 4.81893$ appear as accumulation points in this scheme. 
Up to now we calculated only the first 4 pairs of zeros with sufficient accuracy [19] but S. Skorokhodov from the "Computing Centre of the Russian Academy of Sciences" calculated much more pairs of zeros with an essentially higher accuracy as he informed me in a nice email with the calculated zeros in the appended file [20] (see also [19]). These zeros agreed with my few in lower accuracy calculated zeros 5 .

\section{Conclusions}

In this article we illustrated the behavior of the zeros for low-order Taylor series approximations of the Xi function $\Xi(z)$ as equivalent to the Riemann zeta function $\zeta(z)$ (in view of their nontrivial zeros) and the same of the modified Bessel functions $\left(\frac{2}{z}\right)^{v} \mathrm{I}_{v}(z)$. By scaling of the variable $z$ in the modified Bessel functions we derived by limiting transition $v \rightarrow \infty$ the Gaussian function proportional to $\exp \left(z^{2}\right)$ (with additional scaling of variable $z$ ). The considerations do not pretend to be a proof of the Riemann hypothesis but support the belief to its validity.

There are some bothering problems connected with the zeros of the Riemann zeta function and of functions $\Xi(z)$ of the kind (2.8) with symmetrical functions $\Omega(u)$ which are monotonically decreasing up to their vanishing on the $u$-axis and which are the differences between strictly monotonically and discontinuously vanishing of these functions. The application of the second mean-value theorem leads to the Bessel function of the kind $\sqrt{\frac{\pi}{2 z}} \mathrm{I}_{\frac{1}{2}}(z)=\frac{\operatorname{sh}(z)}{\mathrm{z}}$ with only zeros on the imaginary axis. One may imagine how the mapping onto these function by the second mean-value theorem transforms continuously functions with zeros only on the imaginary axis to the mentioned Bessel function but it is difficult to imagine how this is implemented in this Bessel function for functions of the basic integral representation (2.8) with additional zeros outside the imaginary axis but in Appendix $\mathbf{A}$ it is shown how this apparent contradiction can be solved by a limiting transition. It seems that the presence of zeros off the imaginary axis is restricted to step-wise discontinuous monotonically decreasing functions with periodic steps where the $\Omega(u)$ function to $\frac{\operatorname{sh}(z)}{z}$ itself is step-wise discontinuous and this vanishes in the limiting transition to step lengths zero. Is the second mean-value theorem applicable to include such cases? We think that it is applicable.

The time is now mature for a final solution of all aspects of the Riemann hypothesis concerning the nontrivial zeros of the Riemann zeta function.

\section{Acknowledgements}

Figure 3, Figure 8, Figure 9 and Figure A1 were made with "Mathematica 10.4" ${ }^{5}$ It seems to be possible that with other now derived representations we can calculate more pairs of zeros than given here. 
in preparation of this article. All other Figures were made years ago (about 2010) by "Mathematica 6" and were now after their translation to "Mathematica 10.4" a little improved in few details.

\section{Conflicts of Interest}

The author declares no conflicts of interest regarding the publication of this paper.

\section{References}

[1] Riemann, B. (1859) Über die Anzahl der Primzahlen unter einer gegebenen Grösse, Monatsber. Akad, Berlin, 671-680.

[2] Edwards, H.M. (1974) Riemann's Zeta Function. Dover, New York.

[3] Borwein, P., Choi, S., Rooney, B. and Weirathmueller, A. (2008) The Riemann Hypothesis. Springer, New York.

[4] Whittaker, E.T. and Watson, G.N. (1927) A Course of Modern Analysis. Cambridge University Press, Cambridge.

[5] Titchmarsh, E.C. (1951) The Theory of the Riemann Zeta-Function. Oxford University Press, Oxford.

[6] Chandrasekharan, K. (1970) Arithmetical Functions. Springer-Verlag, Berlin.

[7] Ivić, A. (1985) The Riemann Zeta-Function. Dover Publications, Mineola.

[8] Apostol, M. (2018) Introduction to Analytic Number Theory. Springer, New York.

[9] Neuberger, J.W., Feiler, C., Maier, H. and Schleich, W.P. (2014) Newton Flow of the Riemann Zeta Function: Separatrices Control the Appearance of Zeros. New Journal of Physics, 16, Article ID: 103023. https://doi.org/10.1088/1367-2630/16/10/103023

[10] Neuberger, J.W., Feiler, C., Maier, H. and Schleich, W.P. (2015) The Riemann Hypothesis Illuminated by the Newton Flow of $\zeta$. Physica Scripta, 90, Article ID: 108015.

[11] Wünsche, A. (2016) Approach to a Proof of the Riemann Hypothesis by the Second Mean-Value Theorem of Calculus, General Mathematics. Advances in Pure Mathematics, 6, 972-1021. https://doi.org/10.4236/apm.2016.613074

[12] Watson, G.N. (1944) A Treatise on the Theory of Bessel Functions. 2nd Edition, Cambridge University Press, Cambridge.

[13] Korenev, B.G. (2002) Introduction into the Heory of Bessel Functions. Taylor and Francis, Oxford.

[14] Courant, R. (1937) Differential and Integral Calculus. Vol. 1, 2nd Edition, Interscience, New York.

[15] Widder, D.V. (1989) Advanced Calculus. 2nd Edition, Dover Publications Inc., New York.

[16] Katsnelson, V. Email from 06.01.2017.

[17] Wünsche, A. (2017) Correction and Supplement to Approach for a Proof of Riemann Hypothesis by Second Mean-Value Theorem. Advances in Pure Mathematics, 7, 263-276. https://doi.org/10.4236/apm.2017.73013

[18] Gélinas, J. (2018) Refutation of a Proof of the Riemann Hypothesis by the Bonnet Mean-Value Theorem. Unpublished. 
[19] Wünsche, A. (2018) Wigner Quasiprobability with an Application to Coherent Phase States. Advances in Pure Mathematics, 8, Article ID: 85652. https://doi.org/10.4236/apm.2018.86034

[20] Skorokhodov, S. (2008) Private Communication 2008 in an Email with Appended File, Essential Parts Given in a Footnote. 


\section{Appendix A: Stepwise Constant Functions $\Omega(u)$ and a}

\section{Further Building Stone for the Proof of the Riemann Hypothesis}

In this Appendix we deal with the general case of monotonically decreasing nonnegative real-valued ladder functions $\Omega(u)$ with equal step lengths which satisfy the condition

$$
0 \leq u_{1} \leq u_{2} \Rightarrow \Omega\left(u_{1}\right) \geq \Omega\left(u_{2}\right), \quad \Omega(\infty)=0,
$$

with small improvements in comparison to [17], in particular, also in the notations.

Thus we consider now the following stepwise constant functions $\Omega(u)$ with equal step lengths $u_{0}$ and with monotonically decreasing discrete amplitude platforms $\Omega\left(\left(n+\frac{1}{2}\right) u_{0}\right),(n=0,1,2, \cdots)$

$$
\Omega(u)=\sum_{n=0}^{\infty} \Omega\left(\left(n+\frac{1}{2}\right) u_{0}\right)\left\{\theta\left(u-n u_{0}\right)-\theta\left(u-(n+1) u_{0}\right)\right\},
$$

where $\theta(x)$ denotes the Heaviside step function. The function $\Xi(z)$ possesses then the form

$$
\begin{aligned}
\Xi(z) & =\int_{0}^{+\infty} \mathrm{d} u \Omega(u) \operatorname{ch}(u z) \\
& =\frac{1}{z} \sum_{n=0}^{\infty} \Omega\left(\left(n+\frac{1}{2}\right) u_{0}\right)\left\{\operatorname{sh}\left((n+1) u_{0} z\right)-\operatorname{sh}\left(n u_{0} z\right)\right\} \\
& =\frac{1}{z} \sum_{n=0}^{\infty}\left\{\Omega\left(\left(n+\frac{1}{2}\right) u_{0}\right)-\Omega\left(\left(n+\frac{3}{2}\right) u_{0}\right)\right\} \operatorname{sh}\left((n+1) u_{0} z\right) .
\end{aligned}
$$

Due to monotonicity all amplitudes $\Omega\left(\left(n+\frac{1}{2}\right) u_{0}\right)-\Omega\left(\left(n+\frac{3}{2}\right) u_{0}\right)$ in front of $\operatorname{sh}\left((n+1) u_{0} z\right)$ are nonnegative. We calculate now these functions more in detail. Using the relation

$$
\operatorname{sh}((n+1) w)=\operatorname{sh}(w) \mathrm{U}_{n}(\operatorname{ch}(w)),
$$

to the Chebyshev polynomial $\mathrm{U}_{n}(w)$ we find from (A.3)

$$
\begin{aligned}
& \Xi(z)=\frac{\operatorname{sh}\left(u_{0} z\right)}{z} \sum_{n=0}^{\infty}\left\{\Omega\left(\left(n+\frac{1}{2}\right) u_{0}\right)-\Omega\left(\left(n+\frac{3}{2}\right) u_{0}\right)\right\} \mathrm{U}_{n}\left(\operatorname{ch}\left(u_{0} z\right)\right) \\
& =\frac{\operatorname{sh}\left(u_{0} z\right)}{z} \sum_{n=0}^{\infty}\left\{\Omega\left(\left(n+\frac{1}{2}\right) u_{0}\right)-\Omega\left(\left(n+\frac{3}{2}\right) u_{0}\right)\right\} \sum_{k=0}^{\left[\frac{n}{2}\right]} \frac{(-1)^{k}(n-k) !}{k !(n-2 k) !}\left(2 \operatorname{ch}\left(u_{0} z\right)\right)^{n-2 k} \\
& =\frac{\operatorname{sh}\left(u_{0} z\right)}{z} \sum_{n=0}^{\infty}\left\{\Omega\left(\left(n+\frac{1}{2}\right) u_{0}\right)-\Omega\left(\left(n+\frac{3}{2}\right) u_{0}\right)\right\} \sum_{l=0}^{n} \operatorname{ch}\left((n-2 l) u_{0} z\right) .
\end{aligned}
$$

The stable zeros on the imaginary axis under variation of $u_{0}$ are determined by $u_{0} y=m \pi,(m= \pm 1, \pm 2, \cdots)$ but due to symmetry $y \leftrightarrow-y$ we discuss sometimes so as if we take into account only positive $y$. We now bring the factor 
which determines these zeros in (A.5) to the left-hand side and consider the zeros of the second factor by its separation in real and imaginary part

$$
\begin{aligned}
\frac{z \Xi(z)}{\operatorname{sh}\left(u_{0} z\right)}= & \sum_{n=0}^{\infty}\left\{\Omega\left(\left(n+\frac{1}{2}\right) u_{0}\right)-\Omega\left(\left(n+\frac{3}{2}\right) u_{0}\right)\right\} \\
& \cdot \sum_{l=0}^{n}\left\{\operatorname{ch}\left((n-2 l) u_{0} x\right) \cos \left((n-2 l) u_{0} y\right)\right. \\
& \left.+i \operatorname{sh}\left((n-2 l) u_{0} x\right) \sin \left((n-2 l) u_{0} y\right)\right\} .
\end{aligned}
$$

If we insert for $y$ any zero $y_{m}$ on the imaginary axis determined by $u_{0} y_{m}=m \pi$ with $m \neq 0$ then the imaginary part in (A.6) vanishes and we find

$$
\begin{aligned}
\frac{(x+\mathrm{i} m \pi) \Xi(x+\mathrm{i} m \pi)}{\operatorname{sh}\left(u_{0}(x+\mathrm{i} m \pi)\right)}= & \sum_{n=0}^{\infty}\left\{\Omega\left(\left(n+\frac{1}{2}\right) u_{0}\right)-\Omega\left(\left(n+\frac{3}{2}\right) u_{0}\right)\right\} \\
& \cdot \sum_{l=0}^{n} \operatorname{ch}\left((n-2 l) u_{0} x\right)(-1)^{(n-2 l) m} .
\end{aligned}
$$

In dependence on the chosen $m$ and the amplitudes $\Omega\left(\left(n+\frac{1}{2}\right) u_{0}\right),(n=0,1,2, \cdots)$ one may solve the equations

$$
\sum_{n=0}^{\infty}\left\{\Omega\left(\left(n+\frac{1}{2}\right) u_{0}\right)-\Omega\left(\left(n+\frac{3}{2}\right) u_{0}\right)\right\} \sum_{l=0}^{n}(-1)^{n m} \operatorname{ch}\left((n-2 l) u_{0} x\right)=0,
$$

and all solutions $x=x_{0}$ provide zeros $z_{0}=x_{0}+\mathrm{i} m \pi$ of $\Xi(z)$ for the Omega function in (A.2) off the imaginary axis. If we here formally insert $m=0$ on the left-hand side of (A.8) we see using $\Omega\left(\left(n+\frac{1}{2}\right) u_{0}\right)-\Omega\left(\left(n+\frac{3}{2}\right) u_{0}\right)>0$ that this left-hand side becomes positive with no solution for $x$ at all. If we make the steps $u_{0}$ smaller then the first zero $z=y_{1}$ on the imaginary axis grows according to $y_{1}=\frac{\pi}{u_{0}}$. For the limiting transition $u_{0} \rightarrow 0$ and $y_{1} \rightarrow \infty$ it is not important that we choose the amplitude differences in the middle between two steps as $\Omega\left(\left(n+\frac{1}{2}\right) u_{0}\right)-\Omega\left(\left(n+\frac{3}{2}\right) u_{0}\right)$ and this could be, e.g. also $\Omega\left(n u_{0}\right)-\Omega\left((n+1) u_{0}\right)$.

In Figure A1 we illustrate this by a stepwise function $\Omega(u)$ of the kind (A.2) which approximates the Omega function (2.14) to the Riemann zeta function. If we now make the length $u_{0}$ of the steps smaller then the first imaginary zero $y_{1}$ of the ladder approximation becomes larger according to $y_{m}=\frac{m \pi}{u_{0}}$ and goes for $u_{0} \rightarrow 0$ to infinity and since any zero $z_{0}=x_{0}+\mathrm{i} y_{m}$ off the imaginary axis must possess an imaginary value which is the same as from zeros of the imaginary axis [11] (see also (6.12)) such values $z_{0}$ also go to infinity in the complex plane. Therefore, in the limiting case when $u_{0} \rightarrow 0$ and the ladder curve approaches the function $\Omega(u)$ exactly their zeros off the imaginary also have to go to infinity and they vanish from the pictures of finite-valued zeros in 

Function $\Omega(\mathrm{u})$ to Riemann zeta function and its stepwise approximation with equal step lengths $u_{0}=0.05$
$\Omega(\mathrm{u})$

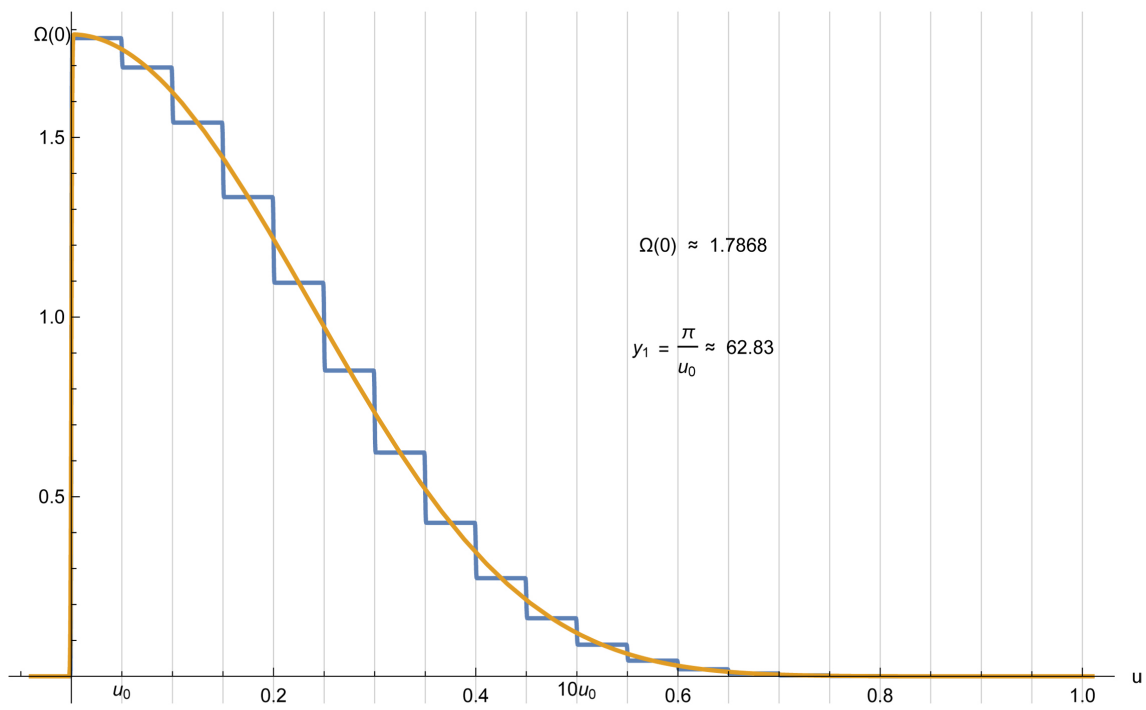

Function $\Omega(\mathrm{u})$ to Riemann zeta function and its stepwise approximation with equal step lengths $u_{0}=0.025$ $\Omega(u)$

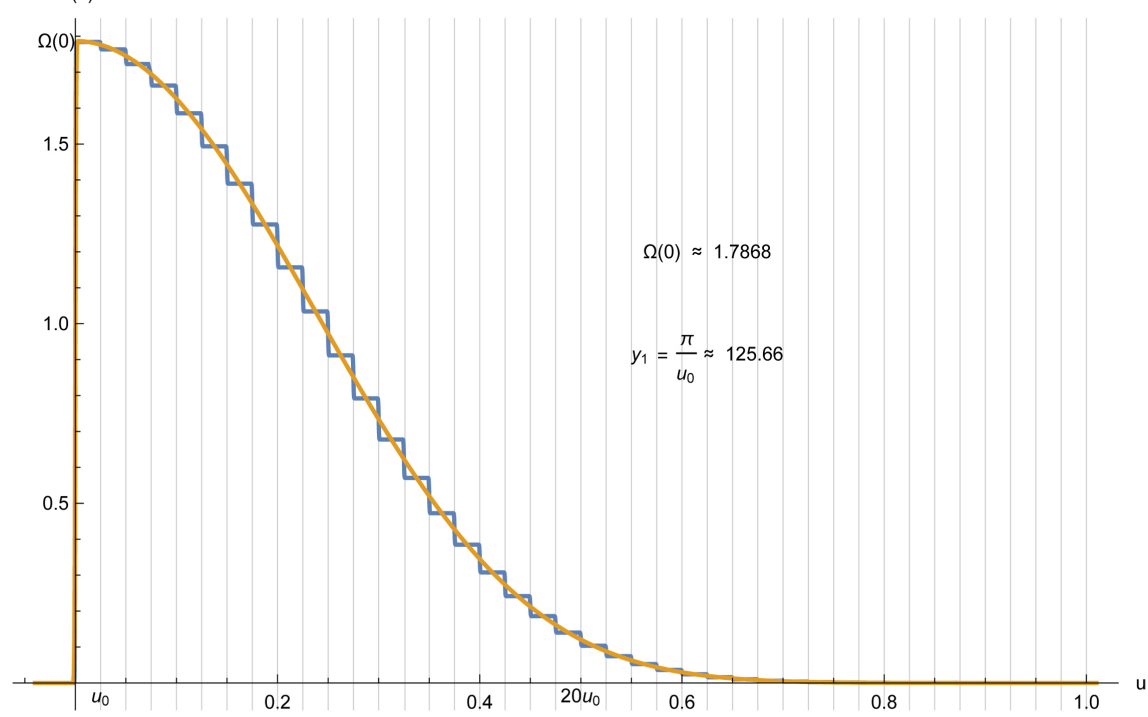

Figure A1. Omega function to Riemann Xi function (2.14) approximated by stepwise constant functions. The steps of equal length $u_{0}$ are here chosen $u_{0}=0.05$ and 0.025 and the first zero $y_{1}$ of the approximation curve of $\Xi(z)$ on the imaginary axis are here at $y_{1}=\frac{\pi}{u_{0}} \approx 62.83$ and $y_{1}=\frac{\pi}{u_{0}} \approx 125.66$. With step length $u_{0} \rightarrow 0$ the imaginary parts of possible zeros off the imaginary axis for the ladder approximations of $\Omega(u)$ to the $\Xi(z)$ functions go to infinity when they approaches to the exact function $\Omega(u)$.

the complex plane. This concerns many functions among them also the $\mathrm{Xi}$ function to the Riemann hypothesis.

For the modified Bessel function where this is known from other more direct proofs, e.g. [12] [13] this is only an affirmation. This also means that for any 
function $\Xi(z)$ with an Omega function $\Omega(u)$ which is different from the described stepwise constant ladder functions with equal step length (A.2) by the same limiting procedure $u_{0} \rightarrow 0$ follows that all zeros off the imaginary axis $y$ are absent in the complex $z$-plane, for example:

$$
\Omega(u)=\left\{\begin{array}{ll}
1-u, & \text { for } 0 \leq u \leq 1 \\
0, & \text { for } u \geq 0
\end{array} \leftrightarrow \quad \Xi(z)=\frac{\operatorname{ch}(z)-1}{z^{2}}=\frac{1}{2}\left(\frac{\operatorname{sh}\left(\frac{z}{2}\right)}{\frac{z}{2}}\right)^{2} .\right.
$$

For this function $\Omega(u)$ it is directly seen that $\Xi(z)$ possesses zeros only on the imaginary axis. Only for the monotonically decreasing ladder functions (A.2) as mentioned the described limiting transition $u_{0} \rightarrow 0$ does not lead to other functions than (A.2) and only these functions may possess zeros outside the imaginary axis which $y$ values in addition have to agree with one of the zeros on the imaginary axis.

We could not find a reason why the Second mean-value theorem should not be applicable to the problem of zeros in the Riemann hypothesis and for other described functions. 